\title{
Real-Time Adaptive Least-Squares Drag Minimization for Performance Adaptive Aeroelastic Wing
}

\author{
Yvonne Ferrier* \\ Stinger Ghaffarian Technologies, Inc., Moffett Field, CA 94035 \\ Nhan Nguyen ${ }^{\dagger}$ \\ NASA Ames Research Center, Moffett Field, CA 94035 \\ Eric Ting $\ddagger$ \\ Stinger Ghaffarian Technologies, Inc., Moffett Field, CA 94035
}

\begin{abstract}
This paper contains a simulation study of a real-time adaptive least-squares drag minimization algorithm for an aeroelastic model of a flexible wing aircraft. The aircraft model is based on the NASA Generic Transport Model (GTM). The wing structures incorporate a novel aerodynamic control surface known as the Variable Camber Continuous Trailing Edge Flap (VCCTEF). The drag minimization algorithm uses the Newton-Raphson method to find the optimal VCCTEF deflections for minimum drag in the context of an altitude-hold flight control mode at cruise conditions. The aerodynamic coefficient parameters used in this optimization method are identified in real-time using Recursive Least Squares (RLS). The results demonstrate the potential of the VCCTEF to improve aerodynamic efficiency for drag minimization for transport aircraft.
\end{abstract}

\section{Introduction}

Air vehicles are typically designed to maintain sufficient structural rigidity for safe load-carrying capacity. Modern engineered materials such as composites have begun to appear in airframe designs that exhibit less structural rigidity while providing the same load-carrying capacity. An example of a light-weight airframe design is the Boeing 787 Dreamliner aircraft, which has more flexible wing structures than older-generation aircraft. This increased structural flexibility afforded by modern materials could be exploited to improve aerodynamic efficiency of future air vehicle concepts.

As the flexibility of aircraft wings increases due to the light-weight composites construction, adverse aerodynamics at off-design can result from aeroelastic deflections. Increased drag, hence more fuel burn, is one such potential consequence. Without means for aeroelastic compensation, the benefit of weight reduction from the use of light-weight material could be negated by sub-optimal aerodynamic performance at off-design flight conditions. Performance Adaptive Aeroelastic Wing (PAAW) technology can potentially address these technical challenges for future flexible wing transports. PAAW technology leverages multidisciplinary solutions to maximize the aerodynamic performance payoff of future adaptive wing design, while simultaneously addressing operational constraints that can prevent optimal aerodynamic performance from being realized.

The Variable Camber Continuous Trailing Edge Flap (VCCTEF), illustrated in Fig. 1, is a possible candidate PAAW concept that has been jointly developed by NASA and Boeing Research \& Technology under the NASA Fixed Wing project. The VCCTEF concept was originally developed under a NASA Innovation Fund study entitled "Elastically Shaped Future Air Vehicle Concept" in 2010. ${ }^{1,2}$ This study examined new concepts that can enable active control of wing aeroelasticity to achieve drag reduction. The results showed that a highly flexible wing could be elastically shaped in-flight by active control of wing twist

*Engineer, Intelligent Systems Division, yvonne.l.ferrier@nasa.gov

†Senior Research Scientist, Associate Fellow AIAA, Intelligent Systems Division, nhan.t.nguyen@nasa.gov

${ }^{\ddagger}$ Engineer, Intelligent Systems Division, eric.b.ting@nasa.gov 
and vertical deflection to improve aerodynamic efficiency during cruise and enhance lift performance during take-off and landing. The VCCTEF concept was developed to address this need ${ }^{1}$ by providing spanwise load tailoring via spanwise flap sections which are joined together by an elastomer material, and chordwise pressure shaping via a variable camber flap having three chordwise segments as shown in Fig. 1. Initial studies indicate that at off-design conditions the VCCTEF system may offer a potential pay-off in drag reduction that could provide significant fuel savings. ${ }^{3}$

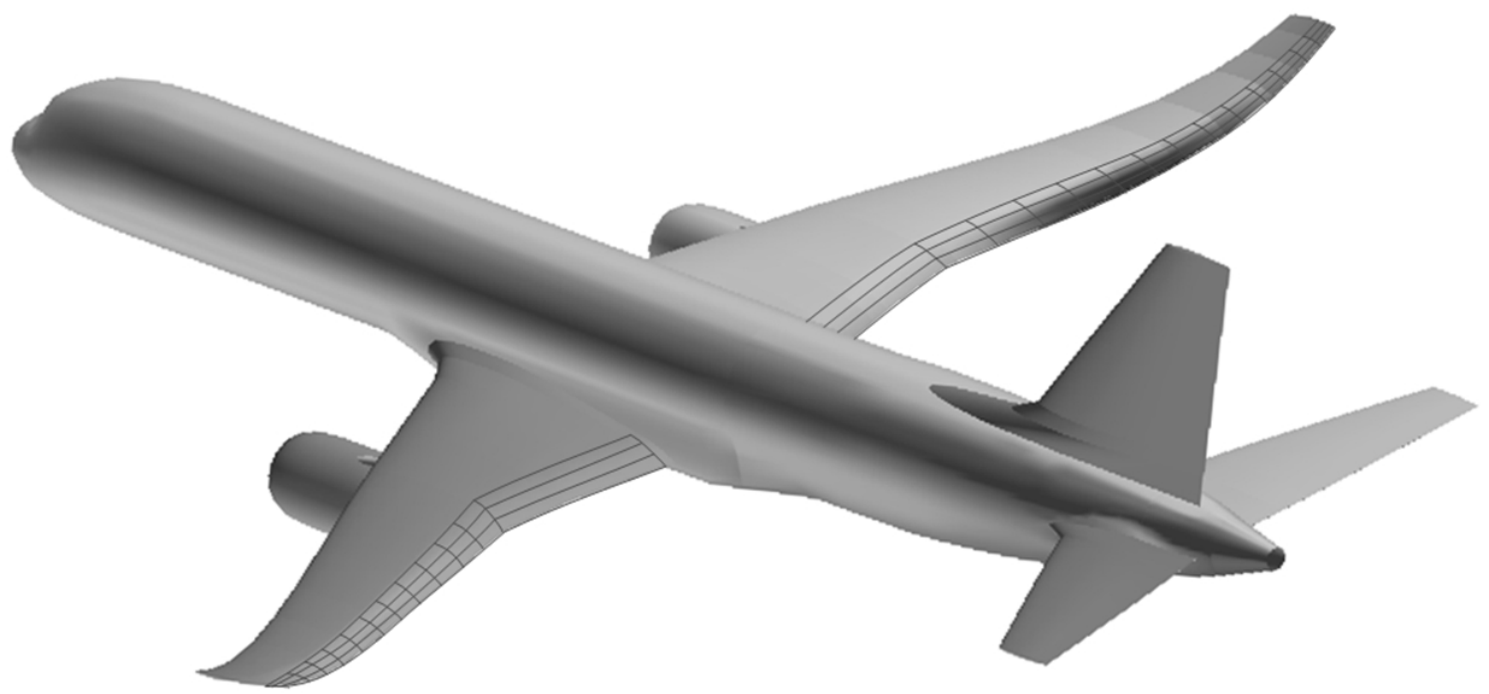

Figure 1. GTM with Variable Camber Continuous Trailing Edge Flap

\section{Problem Statement}

This paper describes the formulation of an aeroelastic model of the GTM with the VCCTEF and the subsequent optimization using a real-time adaptive drag minimization algorithm for an altitude-hold flight control mode at cruise for symmetric flight conditions. In symmetric flight conditions the aircraft is in a wing-level flight and its center of gravity moves only in a vertical plane.

The GTM aircraft model used in this study represents one of the most common types of transport aircraft in the commercial aviation sector that provides short-to-medium range passenger carrying capabilities. Design cruise for this study is assumed to be at $M=0.8$, an altitude of $35,000 \mathrm{ft}, C_{L}=0.4595$, and $50 \%$ fuel load.

The wing planform of the GTM incorporates a VCCTEF layout. The layout of the VCCTEF on the wings used in this study is shown in Fig. 2. The flap system is made up of 19 individual spanwise sections which enable different flap settings at each spanwise position; 15 sections attached to the outer wing and 4 sections attached to the inner wing, as shown in Fig. 2. This results in the ability to control the wing twist shape as a function of span. Changing the wing twist provides the possibility to minimize drag for a specific lift at any aircraft gross weight or mission segment. Wing twist on traditional commercial transport designs is dictated by the aeroelastic deflection of a fixed "jig twist" shape applied at manufacture. The design of this jig twist is set for one cruise configuration, typically for a $50 \%$ fuel loading or mid-point on the gross weight schedule. The VCCTEF offers different wing twist settings, hence different spanwise loadings, for each gross weight condition and also different settings for climb, cruise, and descent, a major factor in obtaining minimum drag at specified lift coefficients.

The multiple spanwise flap sections are connected with a flexible covering and form a continuous trailing edge when the flap sections are deflected. The flexible covering between the flaps sections is an elastomer material and is illustrated in black in Fig. 2. This continuous trailing edge flap is used to optimize the spanwise lift distribution to improve aerodynamic efficiency. An aircraft wing equipped with the VCCTEF can be shaped adaptively to attain better aerodynamic performance throughout a flight envelope. Furthermore, the continuous trailing edge would mitigate strong vortices which otherwise would have formed at the conventional flap discontinuity in the trailing edge region. By mitigating strong vortex formation, viscous 
drag losses as well as acoustic noise from turbulence could be attenuated.

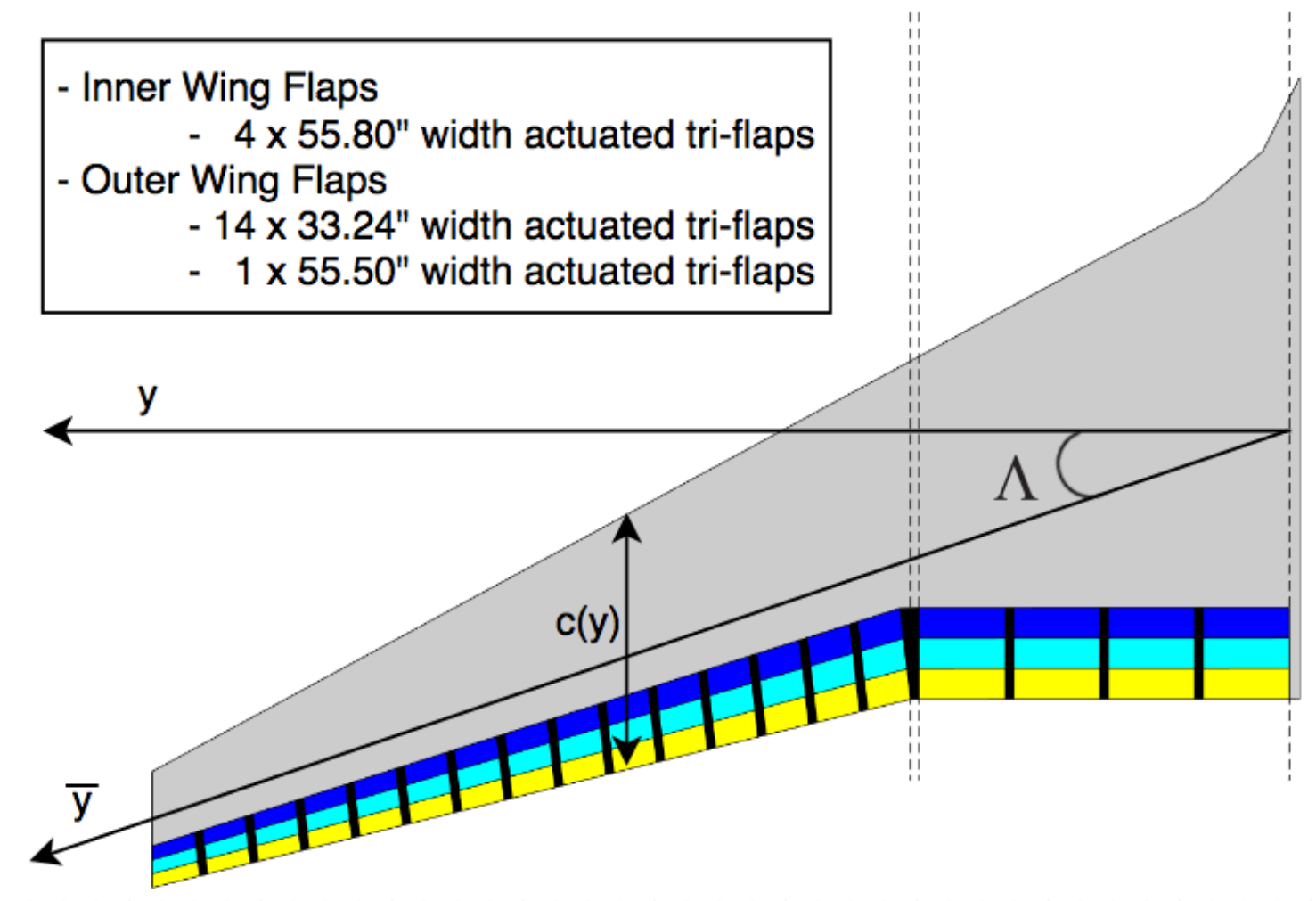

Figure 2. GTM Wing configured with VCCTEF

The spanwise flap sections are restricted in their movement due to structural properties between adjoint sections. To model the candidate optimal shape of the individual flap sections, this study uses a parametrization of the flap deflections using a shape function in the form of a fifth-degree Chebyshev polynomial, given by

$$
\delta_{i, 3}=\sum_{n=0}^{N} a_{n} T_{n}(x)
$$

where $T_{0}(x)=1, T_{1}(x)=x, T_{2}(x)=2 x^{2}-1, T_{3}(x)=4 x^{3}-3 x, T_{4}(x)=8 x^{4}-8 x^{2}-1, T_{5}(x)=$ $16 x^{5}-8 x^{4}-16 x^{3}-8 x^{2}-2 x-1$, and

$$
x=\frac{y_{i}}{L}
$$

with $y_{i}$ as the mid-point of the location of the $i$-th flap section numbered from root to tip and $L$ as the length of half a wing span. This parametrization results in six design variables (DVs) for the drag minimization problem $\left(a_{0}, a_{1}, a_{2}, a_{3}, a_{4}, a_{5}\right)$.

Each flap section of the VCCTEF is comprised of three chordwise segments of equal chord length, as shown in Fig. 3. These three chordwise flap segments can be actuated individually in unison when a flap deflection command is given. By varying the deflections of the individual chordwise flap segments, any camber surface can be created to achieve a desired aerodynamic performance. In this study the segments are coupled according to a circular-arc camber shape, as follows

$$
\begin{aligned}
& \delta_{i, 1}=\frac{1}{3} \delta_{i, 3} \\
& \delta_{i, 2}=\frac{2}{3} \delta_{i, 3}
\end{aligned}
$$

This relation allows the drag minimization algorithm to only consider the deflection angle of the third chordwise flap segment, $\delta_{i, 3}$, since the other two segments will follow accordingly. In a previous study, it was found 
that the circular-arc camber provides one of the best aerodynamic performance among the different camber configurations investigated using OVERFLOW CFD. ${ }^{4}$ The deflection angle of each individual chordwise segment is measured relative to each hinge and the undeflected trailing edge as shown in Fig. 3.

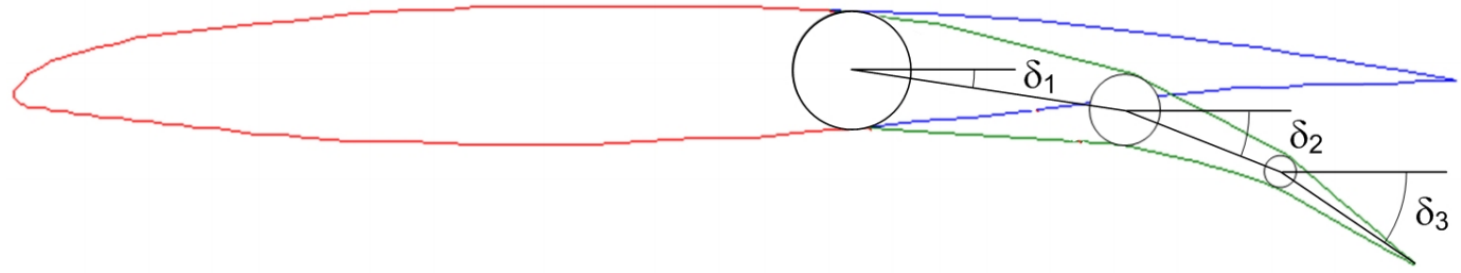

Figure 3. Three-Segment Variable Camber Continuous Trailing Edge Flap

In this study, the drag minimization will not only consider the six DV's of the VCCTEF, but will also take the relating variables into account, namely angle of attack $\alpha$, wing tip bending $w_{t}$, and wing tip twist $\theta_{t}$. The wing tip bending and twist represent the aeroelastic states of the bending $(W)$ and torsion $(\Theta)$ deflections. In this study, the motion of the VCCTEF is assumed to be slow due to the slow response of the Shape Memory Alloy (SMA) actuators. Therefore, the aircraft state variables are also assumed to change slowly. As a result, the coupling of the flight dynamic and aeroservoelastic model is performed under the assumption of quasi-steady aerodynamics. The bending and torsion deflections are expressed as

$$
\begin{aligned}
& W(\bar{y}, t)=\Phi(\bar{y}) w_{t}(t) \\
& \Theta(\bar{y}, t)=\Psi(\bar{y}) \theta_{t}(t)
\end{aligned}
$$

where $\Phi(\bar{y})$ and $\Psi(\bar{y})$ are the mode shape functions of the quasi-steady wing bending and torsion, respectively, and $w_{t}(t)$ and $\theta_{t}(t)$ are the wing tip bending deflection and twist (positive nose down), respectively. The elastic axis, $\bar{y}$, is swept back from the y-axis normal to the flow direction by a sweep angle $\Lambda$ and is illustrated in Fig. 2.

An altitude-hold flight control mode is designed to trim the aircraft at the design cruise altitude of $35000 \mathrm{ft}$. The elevator deflection, $\delta_{e}$, that is required for the altitude-hold flight control mode, will also be taken into consideration in the drag minimization algorithm.

The real-time adaptive drag minimization requires the aerodynamic coefficients to be estimated in flight. A Recursive Least Squares (RLS) identification method is built to estimate the aerodynamic coefficients and derivatives. In this study, an important assumption in the RLS identification approach is that the Mach number does not vary significantly. This assumption is reasonable, since in this study the altitude hold mode ensures that the height does not change noticeably. Furthermore, the airspeed is kept nearly constant by setting it equal to drag.

A simulation study is conducted to assess the real-time adaptive drag minimization strategy.

\section{Model Development}

This section describes the static aeroelastic model for the GTM. The model is constructed from a Galerkin's solution coupled with a vortex-lattice aerodynamic solution through an automatic geometry generation tool.

Furthermore, the modeling of the aerodynamic coefficients and the truth model is discussed. Lastly, the coupled aeroelastic-flight dynamics equations used for the simulation are presented.

\section{A. Aerodynamic Model}

This study uses the Athena Vortex Lattice method (AVL) ${ }^{5}$ as aircraft aerodynamic modeling tool. This method is based on thin wing aerodynamic theory using horseshoe vortex computations. The AVL method does not compute the viscous and wave drag components. The focus of this study is on drag minimization 
and not so much on the actual drag values. The effects of viscous and wave drag do not necessarily alter the adaptive drag minimization approach. Viscous skin friction drag, however, is added to the drag model.

The aerodynamic model is illustrated in Fig. 4. The AVL tool does not include the effect of aeroelasticity and the VCCTEF. For this reason, a deformer is used to change the aircraft geometry based on the aeroelasticity and VCCTEF deflections. The deformer provides the ability to modify the wash-out twist angle of the wing sections to model the effect of aeroelasticity. The aeroelastic wash-out twist is calculated as

$$
\xi_{\text {aeroelastic }}=\Psi(\bar{y}) \theta_{t} \cos \Lambda-\frac{d \Phi(\bar{y})}{d \bar{y}} w_{t} \sin \Lambda
$$

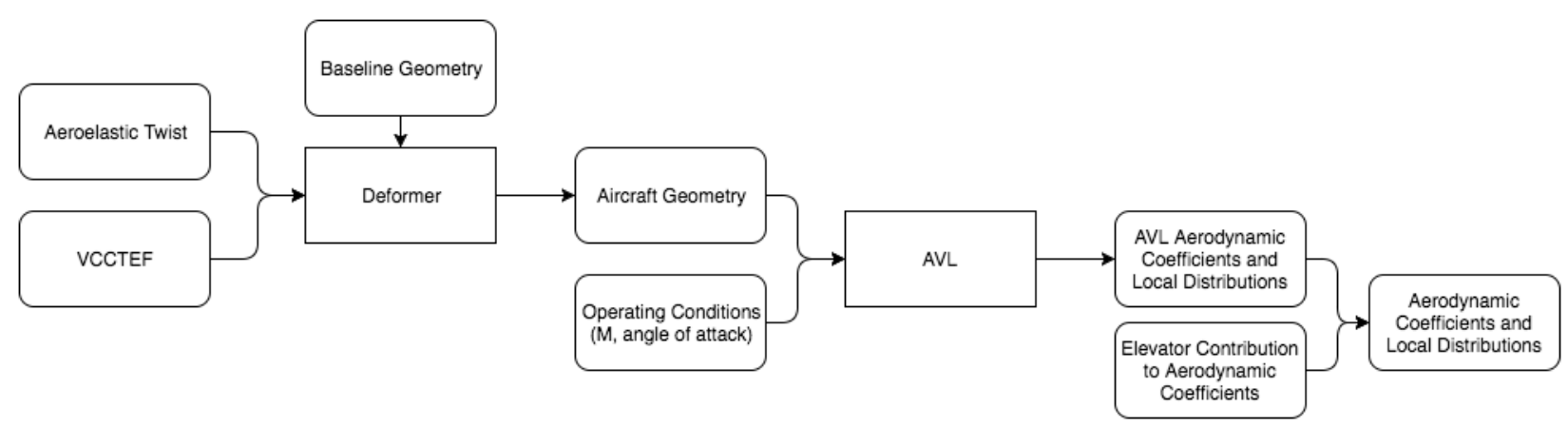

Figure 4. Aerodynamic Model

In this study, the aerodynamic coefficients $\left(C_{D}, C_{L}, C_{m}\right)$ are modeled with respect to the following variables: angle of attack $\alpha$, wing tip bending $w_{t}$, wing tip twist $\theta_{t}$, the Chebyshev coefficients $a_{1 \ldots 5}$, and the elevator deflection $\delta_{e}$.

The drag coefficient $C_{D}$ is approximated to have a quadratic relation between the optimization variables according to

$$
\begin{aligned}
& C_{D}=C_{D_{0}}+C_{D_{\alpha}} \alpha+C_{D_{w_{t}}} w_{t}+C_{D_{\theta_{t}}} \theta_{t}+C_{D_{\delta_{e}}} \delta_{e}+C_{D_{\boldsymbol{a}}} \boldsymbol{a}+C_{D_{\alpha w_{t}}} \alpha w_{t}+C_{D_{\alpha \theta_{t}}} \alpha \theta_{t} \\
& +C_{D_{\alpha \delta_{e}}} \alpha \delta_{e}+C_{D_{\alpha \boldsymbol{a}}} \alpha \boldsymbol{a}+C_{D_{w_{t} \theta_{t}}} w_{t} \theta_{t}+C_{D_{w_{t} \delta_{e}}} w_{t} \delta_{e}+C_{D_{w_{t} \boldsymbol{a}}} w_{t} \boldsymbol{a}+C_{D_{\theta_{t} \delta_{e}}} \theta_{t} \delta_{e} \\
& +C_{D_{\theta_{t} \boldsymbol{a}}} \theta_{t} \boldsymbol{a}+C_{D_{\delta_{e} \boldsymbol{a}}} \delta_{e} \boldsymbol{a}+C_{D_{\alpha^{2}}} \alpha^{2}+C_{D_{w_{t}^{2}}} w_{t}^{2}+C_{D_{\theta_{t}^{2}}} \theta_{t}^{2}+C_{D_{\delta_{e}^{2}}} \delta_{e}^{2}+\boldsymbol{a}^{T} C_{D_{\boldsymbol{a}^{2}}} \boldsymbol{a}
\end{aligned}
$$

where $\boldsymbol{a}=\left[\begin{array}{llllll}a_{0} & a_{1} & a_{2} & a_{3} & a_{4} & a_{5}\end{array}\right]^{T}$ is the vector of the Chebyshev coefficients.

The lift $\left(C_{L}\right)$ and moment $\left(C_{m}\right)$ coefficients are modeled linearly by

$$
\begin{gathered}
C_{L}=C_{L_{0}}+C_{L_{\alpha}} \alpha+C_{L_{w_{t}}} w_{t}+C_{L_{\theta_{t}}} \theta_{t}+C_{L_{\delta_{e}}} \delta_{e}+C_{L_{a}} \boldsymbol{a} \\
C_{m}=C_{m_{0}}+C_{m_{\alpha}} \alpha+C_{m_{w_{t}}} w_{t}+C_{m_{\theta_{t}}} \theta_{t}+C_{m \delta_{e}} \delta_{e}+C_{m_{\boldsymbol{a}}} \boldsymbol{a}
\end{gathered}
$$

The AVL tool does not incorporate the effect of the elevator on the aerodynamic coefficients. For this reason, the elevator contributions are calculated separately, according to

$$
\begin{aligned}
C_{D_{\delta_{e}}} & =\frac{d C_{D_{h}}}{d \delta_{e}} \eta \frac{S_{h}}{S} \\
C_{L_{\delta_{e}}} & =\frac{d C_{L_{h}}}{d \delta_{e}} \eta \frac{S_{h}}{S} \\
C_{m_{\delta_{e}}} & =-\frac{d C_{L_{h}}}{d \delta_{e}} \eta V_{h}
\end{aligned}
$$

where $\frac{d C_{L_{h}}}{d \delta_{e}}$ and $\frac{d C_{D_{h}}}{d \delta_{e}}$ are the elevator drag sensitivity and elevator lift effectiveness, respectively, $\eta$ is the horizontal tail efficiency, $S_{h}$ is the horizontal tail surface, $S$ is the wing surface, and $V_{h}$ is the horizontal tail volume ratio. 
Since $C_{D}$ is modeled as a quadratic function, the quadratic and coupled elevator terms $\left(C_{D_{\delta_{e}^{2}}}, C_{D_{\delta_{e} \mathrm{a}}}\right.$, $\left.C_{D_{\alpha \delta_{e}}}, C_{D_{w_{t} \delta_{e}}}, C_{D_{\theta_{t} \delta_{e}}}\right)$ are also included in the aerodynamic model. These coupled terms are calculated using the following derivation for drag

$$
C_{D}=C_{D_{0}}+K C_{L}^{2}=C_{D_{0}}+K\left(C_{L_{0}}+C_{L_{\alpha}} \alpha+C_{L_{w_{t}}} w_{t}+C_{L_{\theta_{t}}} \theta_{t}+C_{L_{\delta_{e}}} \delta_{e}+C_{L_{\boldsymbol{a}}} \boldsymbol{a}\right)^{2}
$$

where $K=\frac{1}{\pi A R \epsilon}$ is the induced drag parameter which is a function of the wing aspect ratio $A R$ and the Oswald's span efficiency $\epsilon$.

Upon expanding

$$
\begin{aligned}
C_{D}=C_{D_{0}} & +K\left(C_{L_{0}}^{2}+2 C_{L_{0}} C_{L_{\alpha}} \alpha+2 C_{L_{0}} C_{L_{w_{t}}} w_{t}+2 C_{L_{0}} C_{L_{\theta_{t}}} \theta_{t}+2 C_{L_{0}} C_{L_{\delta_{e}}} \delta_{e}+2 C_{L_{0}} C_{L_{\boldsymbol{a}}} \boldsymbol{a}\right. \\
& +2 C_{L_{\alpha}} C_{L_{w_{t}}} \alpha w_{t}+2 C_{L_{\alpha}} C_{L_{\theta_{t}}} \alpha \theta_{t}+2 C_{L_{\alpha}} C_{L_{\delta_{e}}} \alpha \delta_{e}+2 C_{L_{\alpha}} C_{L_{\boldsymbol{a}}} \alpha \boldsymbol{a}+2 C_{L_{w_{t}}} C_{L_{\theta_{t}}} w_{t} \theta_{t} \\
+ & 2 C_{L_{w_{t}}} C_{L_{\delta_{e}}} w_{t} \delta_{e}+2 C_{L_{w_{t}}} C_{L_{\boldsymbol{a}}} w_{t} \boldsymbol{a}+2 C_{L_{\theta_{t}}} C_{L_{\delta_{e}}} \theta_{t} \delta_{e}+2 C_{L_{\theta_{t}}} C_{L_{\boldsymbol{a}}} \theta_{t} \boldsymbol{a}+2 C_{L_{\delta_{e}}} C_{L_{\boldsymbol{a}}} \delta_{e} \boldsymbol{a} \\
& \left.+C_{L_{\alpha}}^{2} \alpha^{2}+C_{L_{w_{t}}}^{2} w_{t}^{2}+C_{L_{\theta_{t}}}^{2} \theta_{t}^{2}+C_{L_{\delta_{e}}}^{2} \delta_{e}^{2}+\boldsymbol{a}^{T} C_{L_{\boldsymbol{a}}}^{2} \boldsymbol{a}\right)
\end{aligned}
$$

Equating Eq. 7 to Eq. 14 results in

$$
\begin{gathered}
C_{D_{\delta_{e}^{2}}}=K C_{L_{\delta_{e}}}^{2} \\
C_{D_{\alpha \delta_{e}}}=2 K C_{L_{\alpha}} C_{L_{\delta_{e}}} \\
C_{D_{w_{t} \delta_{e}}}=2 K C_{L_{w_{t}}} C_{L_{\delta_{e}}} \\
C_{D_{\theta_{t} \delta_{e}}}=2 K C_{L_{\theta_{t}}} C_{L_{\delta_{e}}} \\
C_{D_{a \delta_{e}}}=2 K C_{L_{a}} C_{L_{\delta_{e}}}
\end{gathered}
$$

The local lift and moment distribution are modeled as cubic spline functions with respect to Mach number $M$, angle of attack $\alpha$, wing tip bending $w_{t}$ and twist $\theta_{t}$. The contribution of the VCCTEF to these spline models is assumed to be linear as

$$
\begin{gathered}
c_{l}=c_{l_{\text {spline }}}\left(M, \alpha, w_{t}, \theta_{t}\right)+c_{l_{a}} \boldsymbol{a} \\
c_{m_{a c}}=c_{m_{\text {spline }}}\left(M, \alpha, w_{t}, \theta_{t}\right)+c_{m_{\boldsymbol{a}}} \boldsymbol{a}
\end{gathered}
$$

The altitude-hold control mode requires the formulation of the linear longitudinal flight dynamic statespace model. For this linear model, the longitudinal aerodynamic forces and moments are calculated as

$$
\begin{aligned}
X & =C_{X} q_{\infty} S \\
Z & =C_{Z} q_{\infty} S \\
M & =C_{m} q_{\infty} S \bar{c}
\end{aligned}
$$

where $X$ is the axial force, $Z$ is the normal force and $M$ is the pitching moment. The axial and normal force coefficients are related to the lift and drag coefficients as

$$
\begin{gathered}
C_{X}=C_{L} \sin \alpha-C_{D} \cos \alpha \\
C_{Z}=-C_{L} \cos \alpha-C_{D} \sin \alpha
\end{gathered}
$$

where, for a small angle of attack $\alpha$, this relation can be expressed as

$$
\begin{aligned}
& C_{X}=-C_{D} \\
& C_{Z}=-C_{L}
\end{aligned}
$$

From the above equations, the effect of the VCCTEF on the longitudinal aerodynamic forces and moments and on the force and moment coefficients can be derived as

$$
\begin{gathered}
X_{\mathbf{a}}=C_{X_{\mathbf{a}}} q_{\infty} S \\
C_{X_{\mathbf{a}}}=-C_{D_{\mathbf{a}}} \\
Z_{\mathbf{a}}=C_{Z_{\mathbf{a}}} q_{\infty} S \\
C_{Z_{\mathbf{a}}}=-C_{L_{\mathbf{a}}} \\
M_{\mathbf{a}}=C_{m_{\mathbf{a}}} q_{\infty} S \bar{c}
\end{gathered}
$$




\section{B. Truth Model}

A truth model is built for the GTM that replaces the AVL model of the GTM, and provides the drag $\left(C_{D}\right)$, lift $\left(C_{L}\right)$, and moment $\left(C_{m}\right)$ coefficients along with the local distributions of lift $\left(c_{l}\right)$ and moment coefficients at the quarter chord $\left(c_{m_{a c}}\right)$. The advantage of the truth model over the AVL model is the significant reduction in computation time during simulations.

For the modeling of the truth model, the aerodynamic coefficients are estimated as a function of their aerodynamic coefficient parameters as in Eqs. 7 - 9. Let $\hat{C}_{D}$ be the estimate of $C_{D}$. This estimate of the drag coefficient can be written as

$$
\hat{C}_{D}=\theta_{D}^{T} \phi_{D}\left(\alpha, w_{t}, \theta_{t}, \delta_{e}, \boldsymbol{a}\right)
$$

with

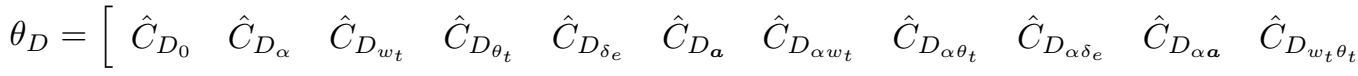

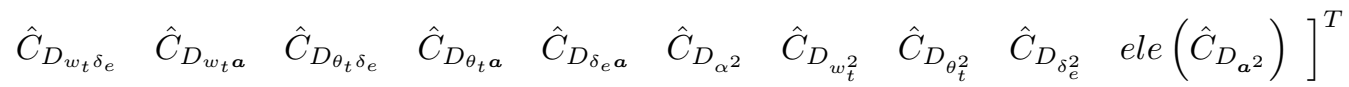

and

$$
\begin{aligned}
\phi_{D}=\left[\begin{array}{llllllllllllllll}
1 & \alpha & w_{t} & \theta_{t} & \delta_{e} & \boldsymbol{a} & \alpha w_{t} & \alpha \theta_{t} & \alpha \delta_{e} & \alpha \boldsymbol{a} & w_{t} \theta_{t} \\
1 & w_{t} \delta_{e} & w_{t} \boldsymbol{a} & \theta_{t} \delta_{e} & \theta_{t} \boldsymbol{a} & \delta_{e} \boldsymbol{a} & \alpha^{2} & w_{t}^{2} & \theta_{t}^{2} & \delta_{e}^{2} & \text { ele }\left(\mathbf{a}^{\top} \mathbf{a}\right)
\end{array}\right]^{T}
\end{aligned}
$$

where ele $(\mathbf{A})$ is the element-wise column vector of $\mathbf{A}$.

The same procedure can be done for the lift coefficient, with $\hat{C}_{L}$ the estimate of $C_{L}$,

$$
\hat{C}_{L}=\theta_{L}^{T} \phi_{L}\left(\alpha, w_{t}, \theta_{t}, \delta_{e}, \boldsymbol{a}\right)
$$

with $\theta_{L}=\left[\begin{array}{llllll}\hat{C}_{L_{0}} & \hat{C}_{L_{\alpha}} & \hat{C}_{L_{w_{t}}} & \hat{C}_{L_{\theta_{t}}} & \hat{C}_{L_{\delta_{e}}} & \hat{C}_{L_{a}}\end{array}\right]^{T}$ and $\phi_{L}=\left[\begin{array}{llllll}1 & \alpha & w_{t} & \theta_{t} & \delta_{e} & \boldsymbol{a}\end{array}\right]^{T}$ and for the moment coefficient, with $\hat{C}_{m}$ the estimate of $C_{m}$,

$$
\hat{C}_{m}=\theta_{m}^{T} \phi_{m}\left(\alpha, w_{t}, \theta_{t}, \delta_{e}, \boldsymbol{a}\right)
$$

with $\theta_{m}=\left[\begin{array}{llllll}\hat{C}_{m_{0}} & \hat{C}_{m_{\alpha}} & \hat{C}_{m_{w_{t}}} & \hat{C}_{m_{\theta_{t}}} & \hat{C}_{m_{\delta_{e}}} & \hat{C}_{m_{\boldsymbol{a}}}\end{array}\right]^{T}$ and $\phi_{m}=\left[\begin{array}{cccccc}1 & \alpha & w_{t} & \theta_{t} & \delta_{e} & \boldsymbol{a}\end{array}\right]^{T}$

The truth model is created by using a batch least squares identification algorithm to define the aerodynamic parameters $\left(\theta_{D}, \theta_{L}\right.$ and $\left.\theta_{m}\right)$, including the influence of the Mach number, but excluding the contribution of the elevator. The truth model for $C_{D}, C_{L}$, and $C_{m}$ is generated by creating a large dataset for different inputs in Mach number, $0.6 \leq M \leq 0.8$, angle of attack, $0^{\circ} \leq \alpha \leq 5^{\circ}$, wing tip bending, $-2 \mathrm{ft} \leq w_{t} \leq 6 \mathrm{ft}$, wing tip twist, $-5^{\circ} \leq \theta_{t} \leq 0^{\circ}$, and VCCTEF deflections, $-5^{\circ} \leq \delta \leq 10^{\circ}$. First, the dataset is separated for the different Mach numbers, and the parameters are estimated according to

$$
\begin{aligned}
\theta_{D} & =\left(A_{D}\right)^{-1} B_{D} \\
\theta_{L} & =\left(A_{L}\right)^{-1} B_{L} \\
\theta_{m} & =\left(A_{m}\right)^{-1} B_{m}
\end{aligned}
$$

with

$$
\begin{aligned}
A_{D} & =\sum \phi_{D}\left(\alpha, w_{t}, \theta_{t}, \boldsymbol{a}\right) \phi_{D}^{T}\left(\alpha, w_{t}, \theta_{t}, \boldsymbol{a}\right) \\
A_{L} & =\sum \phi_{L}\left(\alpha, w_{t}, \theta_{t}, \boldsymbol{a}\right) \phi_{L}^{T}\left(\alpha, w_{t}, \theta_{t}, \boldsymbol{a}\right) \\
A_{m} & =\sum \phi_{m}\left(\alpha, w_{t}, \theta_{t}, \boldsymbol{a}\right) \phi_{m}^{T}\left(\alpha, w_{t}, \theta_{t}, \boldsymbol{a}\right)
\end{aligned}
$$

and 


$$
\begin{aligned}
B_{D} & =\sum \phi_{D}\left(\alpha, w_{t}, \theta_{t}, \boldsymbol{a}\right) C_{D} \\
B_{L} & =\sum \phi_{L}\left(\alpha, w_{t}, \theta_{t}, \boldsymbol{a}\right) C_{L} \\
B_{m} & =\sum \phi_{m}\left(\alpha, w_{t}, \theta_{t}, \boldsymbol{a}\right) C_{m}
\end{aligned}
$$

The next step is to couple the Mach number into the truth model. This is done by estimating the parameters that relate the Mach number to the lift, moment, and drag parameters. In general, the compressibility effect on aerodynamics can be described by the Prandtl-Glauert transformation rule for subsonic flow. For simplicity, the relation with the Mach number is assumed to be cubic, as can be seen in Eq. 48. The Mach parameters are also calculated using a batch least squares identification algorithm, with

$$
\begin{gathered}
\phi_{M}=\left[\begin{array}{c}
1 \\
M \\
M^{2} \\
M^{3}
\end{array}\right] \\
A_{M}=\sum \phi_{M} \phi_{M}^{T} \\
B_{D_{M}}=\sum \phi_{M} \theta_{D} \\
B_{L_{M}}=\sum \phi_{M} \theta_{L} \\
B_{m_{M}}=\sum \phi_{M} \theta_{m} \\
a_{D}=\left(A_{M}\right)^{-1} B_{D_{M}} \\
a_{L}=\left(A_{M}\right)^{-1} B_{L_{M}} \\
a_{m}=\left(A_{M}\right)^{-1} B_{m_{M}}
\end{gathered}
$$

Finally, the truth model algorithm is built upon these estimated aerodynamic and Mach parameters with the following two steps:

1.

$$
\theta_{D}=\phi_{M}^{T} a_{D} \quad \theta_{L}=\phi_{M}^{T} a_{L} \quad \theta_{m}=\phi_{M}^{T} a_{m}
$$

2.

$$
C_{D_{\text {truthmodel }}}=\phi_{D}^{T} \theta_{D} \quad C_{L_{\text {truthmodel }}}=\phi_{L}^{T} \theta_{L} \quad C_{m_{\text {truthmodel }}}=\phi_{m}^{T} \theta_{m}
$$

For the cubic spline modeling of the local lift and moment distributions, the truth model is built using the built-in Matlab function interpn. Because this spline modeling function requires significant computation time, the data set is narrowed down to a subset $\left(0.75 \leq M \leq 0.85,-1^{\circ} \leq \alpha \leq 3^{\circ}\right.$, $2 \mathrm{ft} \leq w_{t} \leq 4 \mathrm{ft}$, $-2^{\circ} \leq \theta_{t} \leq 0^{\circ}$.

In order to verify the accuracy of the truth model, the root-mean-square error (RMSE) and mean percent error (MPE) for the aerodynamic parameters and local distributions are calculated with Eq. 58 and Eq. 59. The results are given in Table 1. From this, we can conclude that the truth model gives an accurate estimation of the aerodynamic parameters.

$$
\begin{gathered}
\operatorname{RMSE}[-]=\sqrt{\frac{\sum_{t=1}^{n}\left(\hat{y}_{t}-y_{t}\right)^{2}}{n}} \\
\operatorname{MPE}[\%]=100 \% \times\left|\frac{\frac{\sum_{t=1}^{n}\left(\hat{y}_{t}-y_{t}\right)}{n}}{\frac{\sum_{t=1}^{n} y_{t}}{n}}\right|
\end{gathered}
$$

where $\hat{y}_{t}$ is the truth model estimate for the parameter, $y_{t}$ the value for that estimate from AVL, and $n$ the total number of comparison data. 


\begin{tabular}{|c|c|c|}
\hline Parameter & RMSE [-] & MPE [\%] \\
\hline \hline$C_{D}$ & $2.4 \cdot 10^{-5}$ & 0.16 \\
\hline$C_{L}$ & $1.0 \cdot 10^{-3}$ & 0.18 \\
\hline$C_{m}$ & $5.5 \cdot 10^{-4}$ & 0.82 \\
\hline$c_{l}$ & $4.4 \cdot 10^{-3}$ & 0.05 \\
\hline$c_{m_{a c}}$ & $1.7 \cdot 10^{-3}$ & 0.13 \\
\hline
\end{tabular}

Table 1. Root-mean-square Error Truth Model

\section{Nonlinear Longitudinal Flight Dynamic Model}

The nonlinear longitudinal flight dynamic model is built for the purpose of the simulation. The relations are given by Eqs. 60 to 64 .

$$
\begin{gathered}
\dot{h}=V \sin \gamma \\
\dot{V}=\frac{T-C_{D} q_{\infty} S-W \sin \gamma}{m} \\
\dot{\gamma}=\frac{C_{L} q_{\infty} S-W \cos \gamma}{m V} \\
\dot{q}=\frac{C_{m} q_{\infty} S \bar{c}+T z_{e}}{I_{y y}} \\
\dot{\theta}=q
\end{gathered}
$$

The drag, lift, and moment coefficients used in the nonlinear flight dynamic equations consist of the truth model coefficients (including the contributions of $M, \alpha, w_{t}, \theta_{t}$ and $\boldsymbol{a}$ ) estimated from AVL and the elevator and pitch rate coefficients according to the following:

$$
\begin{aligned}
& C_{D}=C_{D_{\text {truthmodel }}}+C_{D_{\text {viscous }}}+C_{D_{\delta_{e}}} \delta_{e}+C_{D_{\alpha \delta_{e}}} \alpha \delta_{e}+C_{D_{w_{t} \delta_{e}}} w_{t} \delta_{e}+C_{D_{\theta_{t} \delta_{e}}} \theta_{t} \delta_{e} \\
& +C_{D_{\boldsymbol{a} \delta_{e}}} \delta_{e} \boldsymbol{a}+C_{D_{\delta_{e}}} \delta_{e}^{2}+C_{D_{q}} \frac{q \bar{c}}{2 V} \\
& C_{L}=C_{L_{\text {truthmodel }}}+C_{L_{\delta_{e}}} \delta_{e}+C_{L_{q}} \frac{q \bar{c}}{2 V} \\
& C_{m}=C_{m_{\text {truthmodel }}}+C_{m_{\delta_{e}}} \delta_{e}+C_{m_{q}} \frac{q \bar{c}}{2 V}
\end{aligned}
$$

where the stability derivatives with respect to the pitch rate are

$$
\begin{gathered}
C_{D_{q}}=2 K \bar{C}_{L} C_{L_{q}} \\
C_{L_{q}}=2 C_{L_{\alpha}}\left(\frac{x_{w, a c}}{\bar{c}}-\frac{x_{c g}}{\bar{c}}\right)+2 C_{L_{h, \alpha}} \eta V_{h} \\
C_{m_{q}}=-2 C_{L_{\alpha}}\left(\frac{x_{c g}}{\bar{c}}-\frac{x_{w, a c}}{\bar{c}}\right)^{2}-2 C_{L_{h, \alpha}} \eta V_{h} \frac{l_{h}}{\bar{c}}
\end{gathered}
$$

with $\bar{C}_{L}$ the trim lift coefficient, $x_{w, a c}$ the location of the aerodynamic center of the wing, $x_{c g}$ the location of the center of gravity, $\bar{c}$ the mean aerodynamic chord, $C_{L_{h, \alpha}}$ the horizontal tail lift derivative with respect to $\alpha, \eta$ the horizontal tail efficiency, $V_{h}$ the horizontal tail volume ratio and $l_{h}$ the moment arm of the horizontal tail. The contributions to the drag coefficient of the quadratic and cross-product terms with respect to the pitch rate $q\left(C_{D_{q^{2}}}, C_{D_{\alpha q}}, C_{D_{w_{t} q}}, C_{D_{\theta_{t} q}}, C_{D_{a_{q}}}, C_{D_{\delta_{e} q}}\right)$ are assumed to be neglible.

A constant viscous drag component equal to 0.01 is assumed in this simulation. 


\section{Coupled Aeroelastic Longitudinal Flight Dynamic Model}

The general aeroelastic equations for a coupled bending-torsion motion of a swept wing are expressed as

$$
\begin{gathered}
\rho A \frac{\partial^{2} W}{\partial t^{2}}+\rho A e_{c g} \frac{\partial^{2} \Theta}{\partial t^{2}}+\frac{\partial^{2}}{\partial \bar{y}^{2}}\left(E I_{y y} \frac{\partial^{2} W}{\partial t^{2}}\right)=\bar{l} \cos \Lambda+\frac{\partial \bar{m}}{\partial \bar{y}} \cos \Lambda \sin \Lambda \\
\rho I_{x x} \frac{\partial^{2} \Theta}{\partial t^{2}}+\rho A e_{c g} \frac{\partial^{2} W}{\partial t^{2}}-\frac{\partial}{\partial \bar{y}}\left(G J \frac{\partial \Theta}{\partial \bar{y}}\right)=\bar{m} \cos ^{2} \Lambda
\end{gathered}
$$

where $e_{c g}$ is the offset of the center of mass from the elastic axis, positive when the center of mass lies forward of the elastic axis and $\Lambda$ is the sweep angle of the elastic axis (see Fig. 2). The unsteady lift force and pitching moment are given by

$$
\begin{aligned}
& \bar{l}=C(k) c_{L_{\alpha}} q_{\infty} c\left(\Theta \cos \Lambda-\frac{\partial W}{\partial \bar{y}}\right.\left.\sin \Lambda+\frac{e_{c} \cos \Lambda}{V_{\infty}} \frac{\partial \Theta}{\partial t}-\frac{1}{V_{\infty}} \frac{\partial W}{\partial t}\right) \\
&+\rho_{\infty} \frac{\pi c^{2}}{4}\left(V_{\infty} \cos \Lambda \frac{\partial \Theta}{\partial t}+e_{m} \cos \Lambda \frac{\partial^{2} \Theta}{\partial t^{2}}-\frac{\partial^{2} W}{\partial t^{2}}\right)+\bar{l}_{r}+c_{L_{\mathbf{a}}} q_{\infty} c \mathbf{a} \quad(73) \\
& \bar{m}=C(k) c_{L_{\alpha}} q_{\infty} c e\left(\Theta \cos \Lambda-\frac{\partial W}{\partial \bar{y}} \sin \Lambda+\frac{e_{c} \cos \Lambda}{V_{\infty}} \frac{\partial \Theta}{\partial t}-\frac{1}{V_{\infty}} \frac{\partial W}{\partial t}\right) \\
&-\rho_{\infty} \frac{\pi c^{2}}{4} e_{m}\left(e_{m} \cos \Lambda \frac{\partial^{2} \Theta}{\partial t^{2}}-\frac{\partial^{2} W}{\partial t^{2}}\right)-\rho_{\infty} \frac{\pi c^{2}}{4} e_{c} V_{\infty} \frac{\partial \Theta}{\partial t} \cos \Lambda-\rho_{\infty} \frac{\pi c^{4}}{128} \frac{\partial^{2} \Theta}{\partial t^{2}} \cos \Lambda+\bar{m}_{r}+\left(c_{m_{\mathbf{a}}}+\frac{e}{c} c_{L_{\mathbf{a}}}\right) q_{\infty} c^{2} \mathbf{a}
\end{aligned}
$$

where $e$ is the location of the elastic axis, $e_{m}$ is the distance between the elastic axis and the mid-chord point, $e_{c}$ is the distance between the elastic axis and three-quarter chord point, and $c$ is the sectional chord length (see Fig. 2), $\bar{l}_{r}$ is the rigid lift contribution and $\bar{m}_{r}$ is the rigid moment contribution. Since this study assumes quasi-steady aerodynamics, the Theodorsen's circulation function $C(k)$ is equal to unity. ${ }^{6}$

For drag minimization, the aircraft is assumed to be in quasi-steady conditions. Therefore, the wing aeroelastic effect is assumed to be governed by mostly the first bending mode and torsion mode. As a result, the aeroelastic bending and torsion equations can be described as functions of only the wing tip bending and wing tip twist as in Eqs. 4 and 5. Using this formulation, the simplified aeroelastic equations (Eqs. 71 and 72) can be modeled numerically according to the Galerkin method as

$$
\left[\mathbf{M}+\mathbf{M}_{a}\right]\left[\begin{array}{c}
\ddot{w}_{t} \\
\ddot{\theta}_{t}
\end{array}\right]+\left[\mathbf{C}+\mathbf{C}_{\mathbf{a}}\right]\left[\begin{array}{c}
\dot{w}_{t} \\
\dot{\theta}_{t}
\end{array}\right]+\left[\mathbf{K}+\mathbf{K}_{\mathbf{a}}\right]\left[\begin{array}{c}
w_{t} \\
\theta_{t}
\end{array}\right]=\mathbf{F}_{r} \mathbf{x}_{r}+\mathbf{F}_{\mathbf{a}} \mathbf{a}
$$

where $\mathbf{K}$ is the generalized structural stiffness matrix, $\mathbf{K}_{\mathbf{a}}$ is the aerodynamic stiffness matrix, $\mathbf{C}$ is the generalized structural damping matrix, $\mathbf{C}_{\mathbf{a}}$ is the aerodynamic damping matrix, $\mathbf{M}$ is the generalized structural mass matrix, $\mathbf{M}_{\mathbf{a}}$ is the aerodynamic mass matrix and where $\mathbf{F}_{r}$ is

$$
\mathbf{F}_{r}=\frac{q_{\infty} \cos \Lambda}{V_{\infty}} \int_{0}^{L}\left[\begin{array}{ccccc}
0 & 0 & V_{\infty} c C(k) c_{L_{\alpha}} \Phi^{T}(\bar{y}) & c^{2}\left(\frac{3}{4}+\frac{x_{L E}}{c}\right) C(k) c_{L_{\alpha}} \Phi^{T}(\bar{y}) & 0 \\
0 & 0 & V_{\infty} c e C(k) c_{L_{\alpha}} \cos \Lambda \Psi(\bar{y}) & c^{2} e\left(\frac{3}{4}+\frac{x_{L E}}{c}\right) \cos \Lambda C(k) c_{L_{\alpha}} \Psi(\bar{y}) & 0
\end{array}\right]
$$

and $\mathbf{F}_{\boldsymbol{a}}$ is

$$
\mathbf{F}_{\boldsymbol{a}}=q_{\infty}\left[\begin{array}{c}
\int_{0}^{L} \Phi^{T}(\bar{y}) c_{L_{\boldsymbol{a}}} c \cos \Lambda d \bar{y} \\
\int_{0}^{L} \Psi^{T}(\bar{y})\left(c_{m_{\boldsymbol{a}}}+\frac{e}{c} c_{L_{\boldsymbol{a}}}\right) c^{2} \cos ^{2} \Lambda d \bar{y}
\end{array}\right]
$$

with $c_{L}$ the sectional lift distribution, $\bar{x}_{L E}$ the location of the leading edge of the wing section relative to the aircraft center of gravity and $e$ the location of the elastic axis.

The state vector for the rigid-body aircraft $\mathbf{x}_{r}$ is 


$$
\mathbf{x}_{r}=\left[\begin{array}{c}
h \\
V \\
\alpha \\
q \\
\theta
\end{array}\right]
$$

\section{Flight Control}

Optimal flight control by means of a linear-quadratic regulator (LQR) is implemented to provide an altitude-hold mode during the drag minimization algorithm simulation. The altitude-hold mode is used to trim the aircraft at the design cruise altitude. This altitude-hold mode minimizes aircraft motion for ride qualities.

Furthermore, the airspeed is set equal to drag to ensure the aircraft stays at a constant Mach number. This is implemented not only for passenger comfort, but also because an important assumption in the drag minimization algorithm is a constant Mach number.

\section{A. Linear Longitudinal Flight Dynamic State-Space Model}

For the purpose of control design, the nonlinear longitudinal flight dynamic model is linearized about a trimmed cruise condition, resulting in a linear longitudinal flight dynamic state-space model of the form

$$
\left[\begin{array}{c}
\Delta \dot{u} \\
\Delta \dot{\alpha} \\
\dot{q} \\
\Delta \dot{\theta}
\end{array}\right]=\left[\begin{array}{cccc}
X_{u} & X_{\alpha} & X_{q} & -g \\
Z_{u} & Z_{\alpha} & Z_{q}+\bar{V} & 0 \\
M_{u} & M_{\alpha} & M_{q} & 0 \\
0 & 0 & 1 & 0
\end{array}\right]\left[\begin{array}{c}
\Delta u \\
\Delta \alpha \\
q \\
\Delta \theta
\end{array}\right]+\left[\begin{array}{cc}
X_{\delta_{e}} & X_{\delta} \\
Z_{\delta_{e}} & Z_{\delta} \\
M_{\delta_{e}} & M_{\delta} \\
0 & 0
\end{array}\right]\left[\begin{array}{c}
\Delta \delta_{e} \\
\delta
\end{array}\right]
$$

where $\delta$ are the flap deflections of the VCCTEF.

For the purpose of the altitude hold mode, this state-space system is augmented with an integral error state of the flight path angle, given by

$$
x_{\gamma}=\int_{0}^{t} \Delta \gamma d \tau
$$

where $\Delta \gamma=\gamma-\gamma_{c}=\Delta \theta-\Delta \alpha-\gamma_{c}$ is the error between the flight path angle and the command signal.

This results in the augmented state-space model of the form

$$
\left[\begin{array}{c}
\Delta \dot{u} \\
\Delta \dot{\alpha} \\
\dot{q} \\
\Delta \dot{\theta} \\
\dot{x}_{\gamma}
\end{array}\right]=\left[\begin{array}{ccccc}
X_{u} & X_{\alpha} & X_{q} & -g & 0 \\
Z_{u} & Z_{\alpha} & Z_{q}+\bar{V} & 0 & 0 \\
M_{u} & M_{\alpha} & M_{q} & 0 & 0 \\
0 & 0 & 1 & 0 & 0 \\
0 & -1 & 0 & 1 & 0
\end{array}\right]\left[\begin{array}{c}
\Delta u \\
\Delta \alpha \\
q \\
\Delta \theta \\
x_{\gamma}
\end{array}\right]+\left[\begin{array}{cc}
X_{\delta_{e}} & X_{\delta} \\
Z_{\delta_{e}} & Z_{\delta} \\
M_{\delta_{e}} & M_{\delta} \\
0 & 0 \\
0 & 0
\end{array}\right]\left[\begin{array}{c}
\Delta \delta_{e} \\
\delta
\end{array}\right]+\left[\begin{array}{c}
0 \\
0 \\
0 \\
0 \\
-\gamma_{c}
\end{array}\right]
$$

\section{B. VCCTEF Virtual Control Variables}

As mentioned before, the VCCTEF deflections, $\delta$, are parametrized as a function of the constants, $a_{0} \cdots 5$, by the 5th-degree Chebyshev polynomial shape function (see Eq. 1). For the altitude hold mode with real-time drag minimization, these constants form a command vector instead of an input vector. The state-space model is rewritten as 


$$
\begin{aligned}
{\left[\begin{array}{c}
\Delta \dot{u} \\
\Delta \dot{\alpha} \\
\dot{q} \\
\Delta \dot{\theta} \\
\dot{x}_{\gamma}
\end{array}\right]=\left[\begin{array}{ccccc}
X_{u} & X_{\alpha} & X_{q} & -g & 0 \\
Z_{u} & Z_{\alpha} & Z_{q}+\bar{V} & 0 & 0 \\
M_{u} & M_{\alpha} & M_{q} & 0 & 0 \\
0 & 0 & 1 & 0 & 0 \\
0 & -1 & 0 & 1 & 0
\end{array}\right]\left[\begin{array}{c}
\Delta u \\
\Delta \alpha \\
q \\
\Delta \theta \\
x_{\gamma}
\end{array}\right] } \\
+ \\
+\left[\begin{array}{c}
X_{\delta_{e}} \\
Z_{\delta_{e}} \\
M_{\delta_{e}} \\
0 \\
0
\end{array}\right] \Delta \delta_{e}+\left[\begin{array}{cccccc}
X_{a_{0}} & X_{a_{1}} & X_{a_{2}} & X_{a_{3}} & X_{4} & X_{a_{5}} \\
Z_{a_{0}} & Z_{a_{1}} & Z_{a_{2}} & Z_{a_{3}} & Z_{a_{4}} & Z_{a_{5}} \\
M_{a_{0}} & M_{a_{1}} & M_{a_{2}} & M_{a_{3}} & M_{a_{4}} & M_{a_{5}} \\
0 & 0 & 0 & 0 & 0 & 0 \\
0 & 0 & 0 & 0 & 0 & 0
\end{array}\right]\left[\begin{array}{c}
a_{0} \\
a_{1} \\
a_{2} \\
a_{3} \\
a_{4} \\
a_{5}
\end{array}\right]+\left[\begin{array}{c}
0 \\
0 \\
0 \\
0 \\
-\gamma_{c}
\end{array}\right]
\end{aligned}
$$

The commands $a_{i}(t), i=1, \ldots, N$, are functions of time prescribed by an input random sample for the RLS identification.

\section{Coupled Flight Dynamic and Aeroservoelastic Model}

A coupled longitudinal flight dynamic and aeroservoelastic (ASE) model of the GTM is developed. It consists of the linear flight dynamic model, a linear aeroelastic model of the flexible wing, and a linear actuator model of the VCCTEF for the aeroelastic states.

Implementation of the aeroelastic variables defined in Eq. 75 into the linear state-space system results in the following model:

$$
\begin{aligned}
& {\left[\begin{array}{c}
\Delta \dot{u} \\
\Delta \dot{\alpha} \\
\dot{q} \\
\Delta \dot{\theta} \\
\dot{x}_{\gamma} \\
\Delta \dot{w}_{t} \\
\Delta \dot{\theta}_{t} \\
\Delta \ddot{w}_{t} \\
\Delta \ddot{\theta}_{t}
\end{array}\right]=\left[\begin{array}{ccccccccc}
X_{u} & X_{\alpha} & X_{q} & -g & 0 & X_{w_{t}} & X_{\theta_{t}} & X_{\dot{w}_{t}} & X_{\dot{\theta}_{t}} \\
Z_{u} & Z_{\alpha} & Z_{q}+\bar{V} & 0 & 0 & Z_{w_{t}} & Z_{\theta_{t}} & Z_{\dot{w}_{t}} & Z_{\dot{\theta}_{t}} \\
M_{u} & M_{\alpha} & M_{q} & 0 & 0 & M_{w_{t}} & M_{\theta_{t}} & M_{\dot{w}_{t}} & M_{\dot{\theta}_{t}} \\
0 & 0 & 1 & 0 & 0 & 0 & 0 & 0 & 0 \\
0 & -1 & 0 & 1 & 0 & 0 & 0 & 0 & 0 \\
0 & 0 & 0 & 0 & 0 & 0 & 0 & 1 & 0 \\
0 & 0 & 0 & 0 & 0 & 0 & 0 & 0 & 1 \\
F_{r_{11}} & F_{r_{12}} & F_{r_{13}} & F_{r_{14}} & F_{r_{15}} & K_{11} & K_{12} & C_{11} & C_{12} \\
F_{r_{21}} & F_{r_{22}} & F_{r_{23}} & F_{r_{24}} & F_{r_{25}} & K_{21} & K_{22} & C_{21} & C_{22}
\end{array}\right]\left[\begin{array}{c}
\Delta u \\
\Delta \alpha \\
q \\
\Delta \theta \\
x_{\gamma} \\
\Delta w_{t} \\
\Delta \theta_{t} \\
\Delta \dot{w}_{t} \\
\Delta \dot{\theta}_{t}
\end{array}\right]} \\
& +\left[\begin{array}{c}
X_{\delta_{e}} \\
Z_{\delta_{e}} \\
M_{\delta_{e}} \\
0 \\
0 \\
0 \\
0 \\
0 \\
0
\end{array}\right] \Delta \delta_{e}+\left[\begin{array}{cccccc}
X_{a_{0}} & X_{a_{1}} & X_{a_{2}} & X_{a_{3}} & X_{a_{4}} & X_{a_{5}} \\
Z_{a_{0}} & Z_{a_{1}} & Z_{a_{2}} & Z_{a_{3}} & Z_{a_{4}} & Z_{a_{5}} \\
M_{a_{0}} & M_{a_{1}} & M_{a_{2}} & M_{a_{3}} & M_{a_{4}} & M_{a_{5}} \\
0 & 0 & 0 & 0 & 0 & 0 \\
0 & 0 & 0 & 0 & 0 & 0 \\
0 & 0 & 0 & 0 & 0 & 0 \\
0 & 0 & 0 & 0 & 0 & 0 \\
F_{a_{10}} & F_{a_{11}} & F_{a_{12}} & F_{a_{13}} & F_{a_{14}} & F_{a_{15}} \\
F_{a_{20}} & F_{a_{21}} & F_{a_{22}} & F_{a_{23}} & F_{a_{24}} & F_{a_{25}}
\end{array}\right]\left[\begin{array}{c}
a_{0} \\
a_{1} \\
a_{2} \\
a_{3} \\
a_{4} \\
a_{5}
\end{array}\right]+\left[\begin{array}{c}
0 \\
0 \\
0 \\
0 \\
-\gamma_{c} \\
0 \\
0 \\
0 \\
0
\end{array}\right]
\end{aligned}
$$

with

$$
\begin{gathered}
F_{r_{i j}}=\left\{\left(\mathbf{M}+\mathbf{M}_{\mathbf{a}}\right)^{-1} \mathbf{F}_{r}\right\}_{i j}, i=1,2 j=1,2,3,4,5 \\
K_{i j}=\left\{-\left(\mathbf{M}+\mathbf{M}_{\mathbf{a}}\right)^{-1}\left(\mathbf{K}+\mathbf{K}_{\mathbf{a}}\right)\right\}_{i j}, i=1,2, j=1,2 \\
C_{i j}=\left\{-\left(\mathbf{M}+\mathbf{M}_{\mathbf{a}}\right)^{-1}\left(\mathbf{C}+\mathbf{C}_{\mathbf{a}}\right)\right\}_{i j}, i=1,2, j=1,2
\end{gathered}
$$




$$
F_{a_{i j}}=\left\{\left(\mathbf{M}+\mathbf{M}_{\mathbf{a}}\right)^{-1} \mathbf{F}_{\mathbf{a}}\right\}_{i j}, i=1,2, j=0,1,2,3,4,5
$$

\section{Optimal Control}

The state-space model from Eq. 83 can written in the form

$$
\dot{x}=A x+B u+z
$$

which results in

$$
\begin{aligned}
& {\left[\begin{array}{c}
\Delta \dot{u} \\
\Delta \dot{\alpha} \\
\dot{q} \\
\Delta \dot{\theta} \\
\dot{x}_{\gamma} \\
\Delta \dot{w}_{t} \\
\Delta \dot{\theta}_{t} \\
\Delta \ddot{w}_{t} \\
\Delta \ddot{\theta}_{t}
\end{array}\right]=\left[\begin{array}{ccccccccc}
X_{u} & X_{\alpha} & X_{q} & -g & 0 & X_{w_{t}} & X_{\theta_{t}} & X_{\dot{w}_{t}} & X_{\dot{\theta}_{t}} \\
Z_{u} & Z_{\alpha} & Z_{q}+\bar{V} & 0 & 0 & Z_{w_{t}} & Z_{\theta_{t}} & Z_{\dot{w}_{t}} & Z_{\dot{\theta}_{t}} \\
M_{u} & M_{\alpha} & M_{q} & 0 & 0 & M_{w_{t}} & M_{\theta_{t}} & M_{\dot{w}_{t}} & M_{\dot{\theta}_{t}} \\
0 & 0 & 1 & 0 & 0 & 0 & 0 & 0 & 0 \\
0 & -1 & 0 & 1 & 0 & 0 & 0 & 0 & 0 \\
0 & 0 & 0 & 0 & 0 & 0 & 0 & 1 & 0 \\
0 & 0 & 0 & 0 & 0 & 0 & 0 & 0 & 1 \\
F_{r_{11}} & F_{r_{12}} & F_{r_{13}} & F_{r_{14}} & F_{r_{15}} & K_{11} & K_{12} & C_{11} & C_{12} \\
F_{r_{21}} & F_{r_{22}} & F_{r_{23}} & F_{r_{24}} & F_{r_{25}} & K_{21} & K_{22} & C_{21} & C_{22}
\end{array}\right]\left[\begin{array}{c}
\Delta u \\
\Delta \alpha \\
q \\
\Delta \theta \\
x_{\gamma} \\
\Delta w_{t} \\
\Delta \theta_{t} \\
\Delta \dot{w}_{t} \\
\Delta \dot{\theta}_{t}
\end{array}\right]} \\
& +\left[\begin{array}{c}
X_{\delta_{e}} \\
Z_{\delta_{e}} \\
M_{\delta_{e}} \\
0 \\
0 \\
0 \\
0 \\
0 \\
0
\end{array}\right] \Delta \delta_{e}+\left[\begin{array}{c}
X_{a_{0}} a_{0}+X_{a_{1}} a_{1}+X_{a_{2}} a_{2}+X_{a_{3} a_{3}}+X_{a_{4} a_{4}}+X_{a_{5}} a_{5} \\
Z_{a_{0}} a_{0}+Z_{a_{1}} a_{1}+Z_{a_{2}} a_{2}+Z_{a_{3}} a_{3}+Z_{a_{4}} a_{4}+Z_{a_{5}} a_{5} \\
M_{a_{0}} a_{0}+M_{a_{1}} a_{1}+M_{a_{2}} a_{2}+M_{a_{3}} a_{3}+M_{a_{4}} a_{4}+M_{a_{5}} a_{5} \\
0 \\
-\gamma_{c} \\
0 \\
0 \\
F_{a_{10}} a_{0}+F_{a_{11}} a_{1}+F_{a_{12}} a_{2}+F_{a_{13}} a_{3}+F_{a_{14}} a_{4}+F_{a_{15}} a_{5} \\
F_{a_{20}} a_{0}+F_{a_{21}} a_{1}+F_{a_{22}} a_{2}+F_{a_{23}} a_{3}+F_{a_{24}} a_{4}+F_{a_{25}} a_{5}
\end{array}\right]
\end{aligned}
$$

The optimal control is designed with the following quadratic cost function

$$
J=\frac{1}{2} \int_{0}^{t_{f}}\left[x^{T} Q x+u^{T} R u\right] d t
$$

where $Q$ and $R$ are positive definite. The first term in the cost function is designed to bound the rigid-body states and the aeroelastic modes. The second term is to minimize the control effort. These two terms form the standard linear-quadratic regulator (LQR) cost function.

The Hamiltonian function of the optimal control problem is defined as

$$
H=\frac{1}{2}\left[x^{T} Q x+u^{T} R u\right]+\lambda^{T}(A x+B u+z)
$$

where $\lambda$ is the adjoint vector.

The adjoint equation is obtained as

$$
\dot{\lambda}=-\frac{\partial H^{T}}{\partial x}=-Q x-A^{T} \lambda
$$

The optimal control is obtained as

$$
\frac{\partial H^{T}}{\partial u}=0=R u+B^{T} \lambda \Rightarrow u=-R^{-1} B^{T} \lambda
$$

To solve for these equations, the adjoint equation and the state equation must be solved for simultaneously along with the optimal control. Let $\lambda=P x+S z$ be a solution of the adjoint vector. Then, the adjoint equation is obtained as 


$$
\dot{P} x+P \dot{x}+\dot{S} x+S \dot{z}=-Q x-A^{T} \lambda=-Q x-A^{T}(P x+S z)
$$

The command flight path angle $\gamma_{c}$ is a constant equal to zero and therefore $\dot{\gamma}_{c}=0$. Let $t_{f} \rightarrow \infty$, then the optimal solution approaches a steady state solution. Therefore, $\dot{P}\left(t_{f}\right)=0$ and $\dot{S}\left(t_{f}\right)=0$. Then, separating terms yields the following expressions

$$
\begin{gathered}
P A+A^{T} P-P B R^{-1} B^{T} P+Q=0 \\
S=\left(P B R^{-1} B^{T}-A^{T}\right)^{-1} P
\end{gathered}
$$

The optimal control is then given by

$$
u=K_{x} x+K_{z} z
$$

where

$$
\begin{aligned}
& K_{x}=-R^{-1} B^{T} P \\
& K_{z}=-R^{-1} B^{T} S
\end{aligned}
$$

and $\mathrm{P}$ is the solution to the Algebraic Ricatti Equation of Eq. 95.

Furthermore, the velocity is kept nearly constant during the simulation by setting the thrust equal to drag with

$$
T=C_{D} q_{\infty} S
$$

\section{Adaptive Drag Minimization}

A real-time adaptive drag minimization algorithm is designed for the GTM with flexible wings and VCCTEF. This drag minimization algorithm consists of two parts: first the aerodynamic coefficient parameters are estimated in real-time using the Recursive Least Squares (RLS) estimation method, and secondly the optimal VCCTEF deflections and elevator deflection $\left(\delta_{e}\right)$ are calculated from a constrained optimization problem using the Newton-Raphson method.

\section{A. Online Parameter Identification}

The drag minimization algorithm uses estimates of the aerodynamic coefficients and aerodynamic coefficients parameters to calculate the optimal configuration. For this reason, the aerodynamic coefficients are modeled as functions of their aerodynamic parameters, as in Eqs. 7 - 9. These aerodynamic parameters $\left(\theta_{C_{D}}, \theta_{C_{L}}, \theta_{C_{m}}\right)$ are approximated online using the RLS.

Let $\tilde{C}_{D}=\hat{C}_{D}-C_{D}$ be the estimation error of $C_{D}, \tilde{C}_{L}=\hat{C}_{L}-C_{L}$ the estimation error of $C_{L}$ and $\tilde{C}_{m}=\hat{C}_{m}-C_{m}$ the estimation error of $C_{m}$. Then we have

$$
\begin{gathered}
\tilde{C}_{D}=\theta_{D}^{T} \phi_{D}\left(\alpha, w_{t}, \theta_{t}, \delta_{e}, \boldsymbol{a}\right)-C_{D} \\
\tilde{C}_{L}=\theta_{L}^{T} \phi_{L}\left(\alpha, w_{t}, \theta_{t}, \delta_{e}, \boldsymbol{a}\right)-C_{L}
\end{gathered}
$$

and

$$
\tilde{C}_{m}=\theta_{m}^{T} \phi_{m}\left(\alpha, w_{t}, \theta_{t}, \delta_{e}, \boldsymbol{a}\right)-C_{m}
$$

To find the drag, lift, and moment coefficient parameters, we seek to minimize the estimation errors $\tilde{C}_{D}$, $\tilde{C}_{L}$ and $\tilde{C}_{m}$ with the following quadratic cost functions according to the RLS

$$
\begin{aligned}
J\left(\theta_{D}\right) & =\frac{1}{2} \int_{0}^{t} \tilde{C}_{D}^{2} d \tau=\frac{1}{2} \int_{0}^{t}\left(\theta_{D}^{T} \phi_{D}\left(\alpha, w_{t}, \theta_{t}, \delta_{e}, \boldsymbol{a}\right)-C_{D}\right)^{2} d \tau \\
J\left(\theta_{L}\right) & =\frac{1}{2} \int_{0}^{t} \tilde{C}_{L}^{2} d \tau=\frac{1}{2} \int_{0}^{t}\left(\theta_{L}^{T} \phi_{L}\left(\alpha, w_{t}, \theta_{t}, \delta_{e}, \boldsymbol{a}\right)-C_{L}\right)^{2} d \tau
\end{aligned}
$$




$$
J\left(\theta_{m}\right)=\frac{1}{2} \int_{0}^{t} \tilde{C}_{m}^{2} d \tau=\frac{1}{2} \int_{0}^{t}\left(\theta_{m}^{T} \phi_{m}\left(\alpha, w_{t}, \theta_{t}, \delta_{e}, \boldsymbol{a}\right)-C_{m}\right)^{2} d \tau
$$

The RLS method is an online parameter estimation method where new incoming data $\left(\phi_{i}\right)$ is used to update an existing model. ${ }^{7}$ The RLS identification approach starts with an initial parameter estimate $\left(\theta_{0}\right)$ and covariance matrix $\left(\varepsilon_{0}\right)$ and updates the model according to the following:

1. Update weighting matrix:

$$
K_{i}=\frac{\varepsilon_{i-1} \phi_{i}}{\lambda+\phi_{i}^{T} \varepsilon_{i-1} \phi_{i}}
$$

2. Prediction-error correction formula:

$$
\theta_{i}=\theta_{i-1}+K_{i}\left(y_{i}-\phi_{i}^{T} \theta_{i-1}\right)
$$

3. Corresponding covariance matrix update:

$$
\varepsilon_{i}=\frac{1}{\lambda}\left(I-\varepsilon_{i-1}\left(\frac{\phi_{i} \phi_{i}^{T}}{\lambda+\phi_{i}^{T} \varepsilon_{i-1} \phi_{i}}\right)\right) \varepsilon_{i-1}
$$

where $\varepsilon$ is the covariance matrix, $y$ is the incoming real aerodynamic coefficient, and $\lambda$ is the forgetting factor, which is set to unity for this study. After a certain number of data inputs, the parameter estimates $\left(\theta_{i}\right)$ should converge toward to the "true" parameter values if the training signal is persistently exciting.

\section{B. Optimization Method}

The drag minimization algorithm calculates the optimal VCCTEF configuration for minimum drag during cruise flight. As seen in Eq. 7, the drag coefficient does not only depend on the VCCTEF deflections, parameterized by the Chebyshev polynomial coefficients $\boldsymbol{a}$, but also on the angle of attack $\alpha$, the wing tip bending $w_{t}$, the wing tip twist $\theta_{t}$, and the elevator deflection $\delta_{e}$. For this reason, all of these variables are considered in the drag minimization algorithm. Furthermore, several constraints are imposed on the optimization problem to ensure meeting specific flight characteristics.

The real-time parameter identification allows the use of the identified aerodynamic coefficient parameters in the drag minimization algorithm. The elevator deflection is also included in the optimization and the effect of the elevator on the aerodynamic coefficients is as shown in Eqs. 10 - 12 and Eqs. 15 - 18.

The objective of the drag minimization algorithm is to minimize the drag coefficient modeled in Eq. 7 . Several flight dynamic constraints are imposed on the optimization problem that reduce the dimension of the optimization space.

The first constraint involves selecting a desired value for the lift coefficient, $C_{L_{\mathrm{opt}}}$. Using the model of $C_{L}$ in Eq. 8, the constraint is imposed as in Eq. 110.

$$
C_{L_{o p t}}-\hat{C}_{L_{0}}-\hat{C}_{L_{\alpha}} \alpha-\hat{C}_{L_{w_{t}}} w_{t}-\hat{C}_{L_{\theta_{t}}} \theta_{t}-\hat{C}_{L_{\delta_{e}}} \delta_{e}-\hat{C}_{L_{\boldsymbol{a}}} \boldsymbol{a}=0
$$

In this study, the desired lift coefficient is equal to the design cruise lift coefficient, $C_{L_{o p t}}=0.4595$.

The second constraint imposes a trim condition of zero pitching moment, according to

$$
\hat{C}_{m} \bar{c}+\hat{C}_{D} z_{e}=0
$$

with $\bar{c}$ the mean aerodynamic chord and $z_{e}$ the distance between the engine thrust line and the center of gravity. In the second constraint, it is assumed that thrust is equal to drag, as in Eq. 100.

The third and fourth constraints are to ensure that the optimal wing tip bending and wing tip twist are related to the optimal angle of attack and optimal VCCTEF deflections. A linear relation between the three variables is established from the aeroelastic equations (see Eqs. 73 and 74), as

$$
\begin{gathered}
w_{t}=w_{t_{0}}+w_{t_{\alpha}} \alpha+w_{t_{a}} \boldsymbol{a} \\
\theta_{t}=\theta_{t_{0}}+\theta_{t_{\alpha}} \alpha+\theta_{t_{\boldsymbol{a}}} \boldsymbol{a}
\end{gathered}
$$

where 


$$
\begin{gathered}
w_{t_{\alpha}}=\left[\begin{array}{ll}
1 & 0
\end{array}\right]\left(\mathbf{K}+\mathbf{K}_{\mathbf{a}}\right)^{-1} \mathbf{F}_{\alpha} \\
\theta_{t_{\alpha}}=\left[\begin{array}{ll}
0 & 1
\end{array}\right]\left(\mathbf{K}+\mathbf{K}_{\mathbf{a}}\right)^{-1} \mathbf{F}_{\alpha} \\
w_{t_{\boldsymbol{a}}}=\left[\begin{array}{ll}
1 & 0
\end{array}\right]\left(\mathbf{K}+\mathbf{K}_{\mathbf{a}}\right)^{-1} q_{\infty} \frac{\mathbf{F}_{\boldsymbol{a}}}{q_{\infty}} \\
\theta_{t_{\boldsymbol{a}}}=\left[\begin{array}{ll}
0 & 1
\end{array}\right]\left(\mathbf{K}+\mathbf{K}_{\mathbf{a}}\right)^{-1} q_{\infty} \frac{\mathbf{F}_{\boldsymbol{a}}}{q_{\infty}}
\end{gathered}
$$

with $\mathbf{F}_{\alpha}$ the generalized aerodynamic force and pitching moment due to angle of attack (see Eq. 118), and $\mathbf{F}_{\boldsymbol{a}}$ the generalized aerodynamic force and pitching moment due to the VCCTEF (see Eq. 77).

$$
\mathrm{F}_{\alpha}=\left[\begin{array}{c}
\int_{0}^{L} \Phi^{T}(x)\left(c_{L_{\alpha}} q_{\infty} c \cos \Lambda d x+\frac{d}{d x} c_{L_{\alpha}} q_{\infty} c e \cos \Lambda \sin \Lambda\right) \\
\int_{0}^{L} \Psi^{T}(x) c_{L_{\alpha}} q_{\infty} c e \cos ^{2} \Lambda d x
\end{array}\right]
$$

This results in the following relations for the third and fourth constraints,

$$
\begin{gathered}
\Delta w_{t}-w_{t_{\alpha}} \Delta \alpha-w_{t_{a}} \boldsymbol{a}=0 \\
\Delta \theta_{t}-\theta_{t_{\alpha}} \Delta \alpha-\theta_{t_{a}} \boldsymbol{a}=0
\end{gathered}
$$

Adding these constraints to the objective of minimizing the drag coefficient results in the following augmented drag minimization cost function,

$$
\begin{aligned}
J\left(\alpha, w_{t}, \theta_{t}, \delta_{e}, \boldsymbol{a}\right)= & \hat{C}_{D}+\lambda_{1}\left(C_{L_{o p t}}-\hat{C}_{L_{0}}-\hat{C}_{L_{\alpha}} \alpha-\hat{C}_{L_{w_{t}}} w_{t}-\hat{C}_{L_{\theta_{t}}} \theta_{t}-\hat{C}_{L_{\delta_{e}}} \delta_{e}-\hat{C}_{L_{\boldsymbol{a}}} \boldsymbol{a}\right) \\
& +\lambda_{2}\left(\hat{C}_{m} \bar{c}+\hat{C}_{D} z_{e}\right)+\lambda_{3}\left(\Delta w_{t}-w_{t_{\alpha}} \Delta \alpha-w_{t_{\boldsymbol{a}}} \boldsymbol{a}\right)+\lambda_{4}\left(\Delta \theta_{t}-\theta_{t_{\alpha}} \Delta \alpha-\theta_{t_{\boldsymbol{a}}} \boldsymbol{a}\right)
\end{aligned}
$$

where $\lambda_{1 \ldots 4}$ are Lagrange multipliers or adjoint variables.

The augmented drag minimization cost function can be solved with the Newton-Raphson method. The Newton-Raphson method finds a solution to a cost function by recursively finding better approximations to the roots of a function $\left(\nabla J\left(\Theta_{n}\right)=0\right)$. The method starts with an initial guess $\left(x_{0}\right)$ for a root of the function. If the function satisfies the assumptions made in the derivation of the formula and the initial guess is close, then a better approximation is calculated with Eq.122. This process is repeated until the design variables converge to a sufficiently accurate solution.

$$
x_{n+1}=x_{n}-\left[\nabla^{2} J\left(x_{n}\right)\right]^{-1} \nabla J\left(\Theta_{n}\right)
$$

where $\nabla J$ is the Jacobian and $\nabla^{2} J\left(x_{n}\right)$ is the Hessian of the cost function with respect to the design variables.

The augmented cost function can be solved with the Newton-Raphson method by rewriting Eq. 121 into the following equations:

$$
\begin{aligned}
& \frac{\partial J}{\partial a_{i}}=\left(\hat{C}_{D_{a_{i}}}+2 \hat{C}_{D_{a_{i}^{2}} a_{i}}+\hat{C}_{D_{a_{i} a} a} \boldsymbol{a}+\hat{C}_{D_{a_{i} \alpha}} \alpha+\hat{C}_{D_{a_{i} w_{t}}} w_{t}+\hat{C}_{D_{a_{i} \theta_{t}}} \theta_{t}+\hat{C}_{D_{a_{i} \delta_{e}}} \delta_{e}\right)\left(1+z_{e} \lambda_{2}\right) \\
& -\lambda_{1} \hat{C}_{L_{a_{i}}}+\lambda_{2} \bar{c} \hat{C}_{m_{a_{i}}}-\lambda_{3} w_{t_{a_{i}}}-\lambda_{4} \theta_{t_{a_{i}}}=0 \\
& \frac{\partial J}{\partial \alpha}=\left(\hat{C}_{D_{\alpha}}+2 \hat{C}_{D_{\alpha^{2}}} \alpha+\hat{C}_{D_{\boldsymbol{a} \alpha}} \boldsymbol{a}+\hat{C}_{D_{\alpha w_{t}}} w_{t}+\hat{C}_{D_{\alpha \theta_{t}}} \theta_{t}+\hat{C}_{D_{\alpha \delta_{e}}} \delta_{e}\right)\left(1+z_{e} \lambda_{2}\right) \\
& -\lambda_{1} \hat{C}_{L_{\alpha}}+\lambda_{2} \bar{c} \hat{C}_{m_{\alpha}}-\lambda_{3} w_{t_{\alpha}}-\lambda_{4} \theta_{t_{\alpha}}=0 \\
& \frac{\partial J}{\partial w_{t}}=\left(\hat{C}_{D_{w_{t}}}+2 \hat{C}_{D_{w_{t}^{2}}} w_{t}+\hat{C}_{D_{\boldsymbol{a} w_{t}}} \boldsymbol{a}+\hat{C}_{D_{\alpha w_{t}}} \alpha+\hat{C}_{D_{w_{t} \theta_{t}}} \theta_{t}+\hat{C}_{D_{w_{t} \delta_{e}}} \delta_{e}\right)\left(1+z_{e} \lambda_{2}\right) \\
& -\lambda_{1} \hat{C}_{L_{w_{t}}}+\lambda_{2} \bar{c} \hat{C}_{m_{w_{t}}}+\lambda_{3}=0
\end{aligned}
$$




$$
\begin{aligned}
& \frac{\partial J}{\partial \theta_{t}}=\left(\hat{C}_{D_{\theta_{t}}}+2 \hat{C}_{D_{\theta_{t}^{2}} \theta_{t}}+\hat{C}_{D_{\boldsymbol{a} \theta_{t}}} \boldsymbol{a}+\hat{C}_{D_{\alpha \theta_{t}}} \alpha+\hat{C}_{D_{w_{t} \theta_{t}}} w_{t}+\hat{C}_{D_{\theta t \delta_{e}}} \delta_{e}\right)\left(1+z_{e} \lambda_{2}\right) \\
& -\lambda_{1} \hat{C}_{L_{\theta_{t}}}+\lambda_{2} \bar{c} \hat{C}_{m_{\theta_{t}}}+\lambda_{4}=0 \\
& \frac{\partial J}{\partial \delta_{e}}=\left(\hat{C}_{D_{\delta_{e}}}+2 \hat{C}_{D_{\delta_{e}^{2}} w_{t}}+\hat{C}_{D_{\boldsymbol{a} \delta_{e}}} \boldsymbol{a}+\hat{C}_{D_{\alpha \delta_{e}}} \alpha+\hat{C}_{D_{w_{t} \delta_{e}}} w_{t}+\hat{C}_{D_{\theta_{t} \delta_{e}}} \theta_{t}\right)\left(1+z_{e} \lambda_{2}\right) \\
& -\lambda_{1} \hat{C}_{L_{\delta_{e}}}+\lambda_{2} \bar{c} \hat{C}_{m_{\delta_{e}}}=0 \\
& \frac{\partial J}{\partial \lambda_{1}}=C_{L_{o p t}}-\hat{C}_{L_{0}}-\hat{C}_{L_{a}} \boldsymbol{a}-\hat{C}_{L_{\alpha}} \alpha-\hat{C}_{L_{w_{t}}} w_{t}-\hat{C}_{L_{\theta_{t}}} \theta_{t}-\hat{C}_{L_{\delta_{e}}} \delta_{e}=0 \\
& \frac{\partial J}{\partial \lambda_{2}}=\hat{C}_{m} \bar{c}+\hat{C}_{D} z_{e}=0 \\
& \frac{\partial J}{\partial \lambda_{3}}=\Delta w_{t}-w_{t_{\alpha}} \Delta \alpha-w_{t_{a}} \boldsymbol{a}=0 \\
& \frac{\partial J}{\partial \lambda_{4}}=\Delta \theta_{t}-\theta_{t_{\alpha}} \Delta \alpha-\theta_{t_{a}} a=0
\end{aligned}
$$

with $i$ referring to the specific Chebyshev coefficient $(i=0, \ldots, 5)$. These equations are solved for the variables $\alpha, w_{t}, \theta_{t}, \delta_{e}$ and $\boldsymbol{a}$.

\section{Iterative Refinement Optimization}

The drag minimization algorithm starts with the excitation of the flight dynamic states by giving bounded randomized VCCTEF deflection inputs to the aircraft model. This excitation allows the RLS parameter estimation to converge to an initial estimate of the aerodynamic parameters. After a certain amount of time, the excitation will be damped out and the VCCTEF will be deflected at the optimal deflection configuration.

In order to verify that the aircraft is in its actual minimum drag configuration, an iterative refinement optimization is performed. The iterative refinement optimization consists of locally perturbing the VCCTEF around the estimated optimal flap deflections, allowing the RLS method to update the estimates of the aerodynamic coefficient parameters. If these parameters are updated to different values, a new optimum could be found. From this we can conclude that the previously found optimum is not the actual minimum drag configuration.

During the iterative refinement optimization, the third segment of the VCCTEF is perturbed by

$$
\delta_{i}=\delta_{\mathrm{opt}}+\Delta \delta_{i}
$$

with $-0.5^{\circ} \leq \Delta \delta_{i} \leq 0.5^{\circ}$.

When a new optimal solution is found, the iterative refinement optimization can be repeated after a certain period of time. For a new iterative refinement optimization to take place, a minimum change criteria has to be obtained by the previous iterative refinement optimization. This criteria is necessary to avoid unneeded flap perturbations when the parameter identification has already converged well enough. For this study, a minimum change criteria of 0.00005 in the calculated optimal value for $C_{D}$ is used.

\section{Simulations}

This section contains the results of a $500-$ sec simulation with a step size of $\Delta t=0.01 \mathrm{~s}$. This simulation is done for the GTM with the incorporated VCCTEF control surfaces.

\section{A. Integration Scheme}

For the simulation, three elementary integration schemes are considered, namely the implicit Euler method (Eq. 133), the explicit Euler method (Eq. 134) and the trapezoidal Euler method (Eq. 135). The implicit Euler method is a first order single-step method and has good stability properties. The explicit Euler method has a conditional numerical stability that requires a small step size, $\Delta t$, which can increase the computation time. The Implicit and Explicit Euler methods are both first-order accurate. The trapezoidal 
Euler method is second-order accurate and thus provides a more accurate approximation than the implicit Euler and explicit Euler methods. The trapezoidal Euler method is used in this study because of its good stability and accuracy properties.

$$
\begin{gathered}
\dot{x}=f(x, t) \rightarrow x_{i+1}=x_{i}+\Delta t f\left(x_{i+1}, t_{i+1}\right) \\
\dot{x}=f(x, t)=x_{i}+\Delta t f\left(x_{i}, t_{i}\right) \\
\dot{x}=f(x, t) \rightarrow x_{i+1}=x_{i}+\frac{\Delta t}{2}\left(f\left(x_{i+1}, t_{i+1}\right)+f\left(x_{i}, t_{i}\right)\right)
\end{gathered}
$$

\section{B. Simulation Loop}

The simulation loop is illustrated in Fig. 5. The simulation begins with the clean wing configuration. The simulation starts with a randomized excitation of the VCCTEF, allowing the RLS identification algorithm to estimate the aerodynamic coefficient parameters. The randomized VCCTEF inputs are constrained to be within $-3.6^{\circ} \leq \delta \leq 7.5^{\circ}$ and to change at a maximum rate of $6^{\circ}$ per seconds. These constraints are necessary to avoid unbounded aircraft motion. The randomized inputs are used to create a time-varying command for the flight control which commands the VCCTEF deflections while changing the elevator to hold the aircraft altitude.

After this parameter identification period when the estimated parameters are deemed convergent, the online optimization is performed. Then, the VCCTEF and elevator are deflected to the calculated optimal values. Iterative refinement optimization takes place at $t=300 \mathrm{~s}$ and can take place every 40 seconds after that if needed. The elevator controller determines the elevator deflection by using optimal control for the altitude-hold mode. The VCCTEF is deflected back to the clean wing configuration after the first iterative refinement optimization. At $t=400$ s the VCCTEF is deflected to the calculated optimal configuration. Deflecting the VCCTEF to the clean wing configuration is done to illustrate the difference between the clean wing and optimal configuration in the simulation results. In real flight, the VCCTEF will be deflected directly to the optimal configuration after each iterative refinement optimization.

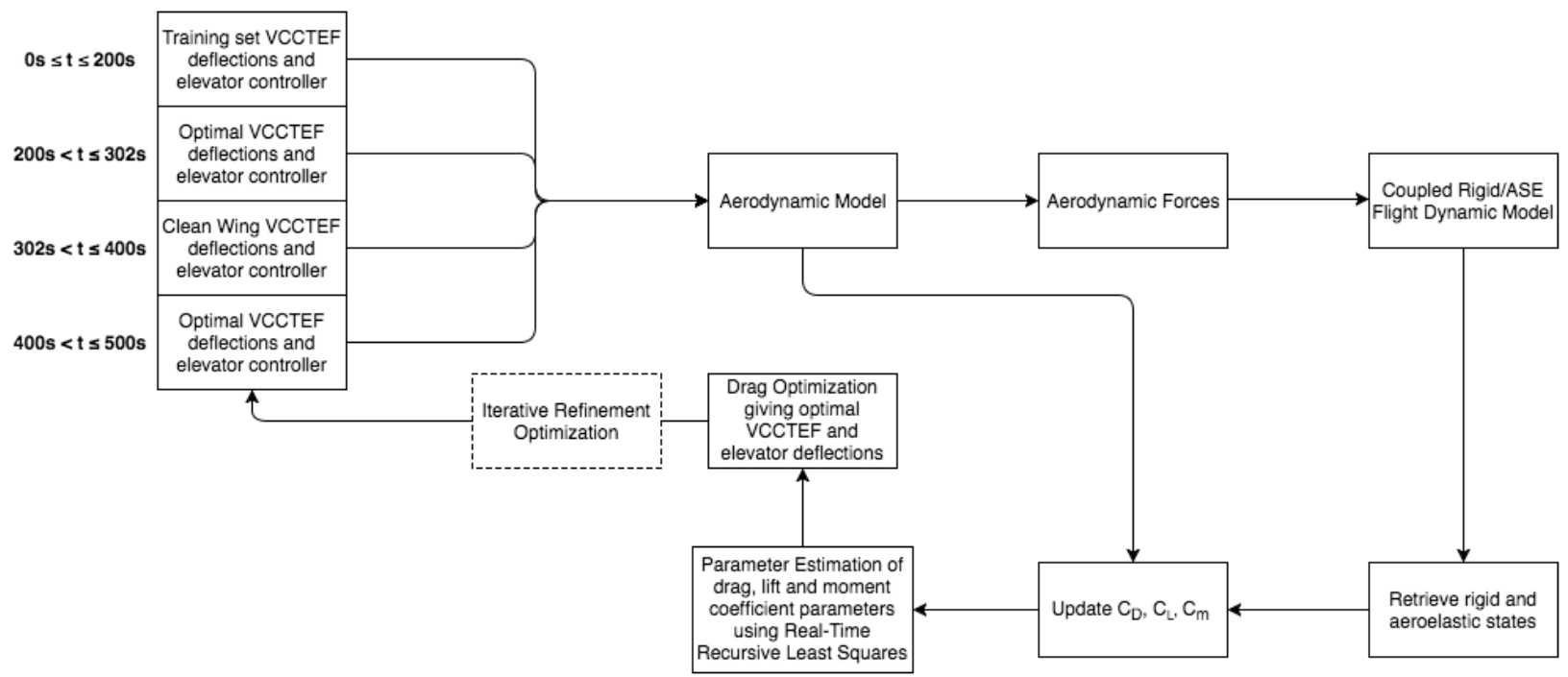

Figure 5. Adaptive Real-time Drag Minimization Control Loop

\section{Simulation Results}

This section contains the results of the simulation of the real-time adaptive drag minimization for the GTM.

\section{Online Parameter Identification}

The RLS identification algorithm starts with a rough estimate of the aerodynamic parameters. In this simulation, the initial drag coefficient parameters are randomized to be approximately $20 \%$ different from 
the true drag parameters, while the initial lift and moment coefficient parameters are randomized to be approximately $10 \%$ different from the true lift and moment parameters. The simulation starts the first 200 seconds with the randomized excitation of the VCCTEF. After 200s, the VCCTEF deflections are set to the calculated optimal values. The RLS identification algorithm continues throughout the simulation.

In Figs. 6 - 8 the convergence of the covariance resetting and the estimation errors $\tilde{C}_{D}, \tilde{C}_{L}$ and $\tilde{C}_{m}$ to zero are illustrated. For visual clarity reasons, the legend in Fig. 6 does not contain all the drag coefficient parameters.

Most covariance resetting values converge to zero in the first 200 seconds, however not all values convergence to zero yet. One can see that during the iterative refinement optimization period (at $t=300 \mathrm{~s}$ ) these values tend to decrease again. This means that for these variables, the identified parameters could still vary significantly for new input data. Longer and more disruptive excitation will allow these covariances to eventually converge to zero. The change in VCCTEF deflections at the iterative refinement optimization $(t=300 \mathrm{~s})$ and at the switch from clean wing to optimal wing configuration $(t=400 \mathrm{~s})$ stimulates the further convergence of the covariance resetting and causes a temporary spike in the estimation errors.

The estimation errors however show that the aerodynamic coefficient estimates are very close to the actual values. As a criteria, it is decided that the estimation error $\tilde{C}_{D}$ should be below 0.0001 . Figure 6 shows that this criteria is achieved.

The updating of the aerodynamic parameters for $\hat{C}_{D}, \hat{C}_{L}$ and $\hat{C}_{m}$ is illustrated in Figs. 9 - 11. For visual clarity reasons, Fig. 9 does not contain all the drag coefficient parameters and only the first 250 seconds of the simulation are illustrated. The final estimated values, actual values and estimation errors for some parameters of $\hat{C}_{D}$ and all parameters for $\hat{C}_{L}$ and $\hat{C}_{m}$ are given in Tables $2-4$.

Comparing Tables $2-4$, it is noteworthy that the RLS identification algorithm is less able to estimate the drag parameters than the lift and moment coefficient parameters. This could be because the number of drag parameters is higher than for the lift and moment parameters, which could increase the difficulty in estimating the influence of each separate variable. Furthermore, the coupled parameters could be harder to identify due to the accumulation of uncertainties in the influence of each separate variable. Moreover, the magnitude of drag parameters are generally small, thus creating a larger uncertainty in the parameter estimation.

It is observed that, for all $\hat{C}_{D}, \hat{C}_{L}$ and $\hat{C}_{m}$, the parameters with respect to wing bending, $w_{t}$, are difficult to estimate accurately. This is probably due to the small value the wing tip bending directly has on the aerodynamic coefficients. The accuracy of these parameters could result in less than optimal solutions. In future research, improved methods will be developed to address this issue.

\begin{tabular}{|c|c|c|c|}
\hline & Final Estimated Value & Truth Model Value & Estimation Error [\%] \\
\hline \hline$C_{D_{0}}$ & 0.0014 & 0.0016 & -8.6 \\
\hline$C_{D_{a_{0}}}$ & 0.0354 & 0.0405 & -12.6 \\
\hline$C_{D_{a_{5}}}$ & 0.0205 & 0.0195 & 5.1 \\
\hline$C_{D_{\alpha}}$ & 0.0908 & 0.0917 & -1.0 \\
\hline$C_{D_{w_{t}}}$ & 0.0001 & -0.0000 & -866.6 \\
\hline$C_{D_{\theta_{t}}}$ & 0.04670 & 0.0404 & 15.6 \\
\hline$C_{D_{a_{0} a_{0}}}$ & 0.2996 & 0.3211 & -6.7 \\
\hline$C_{D_{a_{0} a_{5}}}$ & 0.1927 & 0.1886 & 2.2 \\
\hline$C_{D_{a_{0} \alpha}}$ & 1.2186 & 1.3997 & -12.9 \\
\hline$C_{D_{a_{0} \theta_{t}}}$ & 0.6426 & 0.7239 & -11.2 \\
\hline
\end{tabular}

Table 2. Drag Coefficient Parameter Estimation Value and Estimation Error

\section{Drag Minimization}

The final results of the drag minimization are given in Table 5. The results are compared to the clean wing configuration. The clean wing configuration corresponds to the trim state with zero VCCTEF deflections. For this simulation, $C_{L_{\mathrm{opt}}}=0.4595$ is used. It can be seen that the drag-optimal configuration with the VCCTEF indeed results in a lower drag for the same lift coefficient compared to the clean wing configuration. 

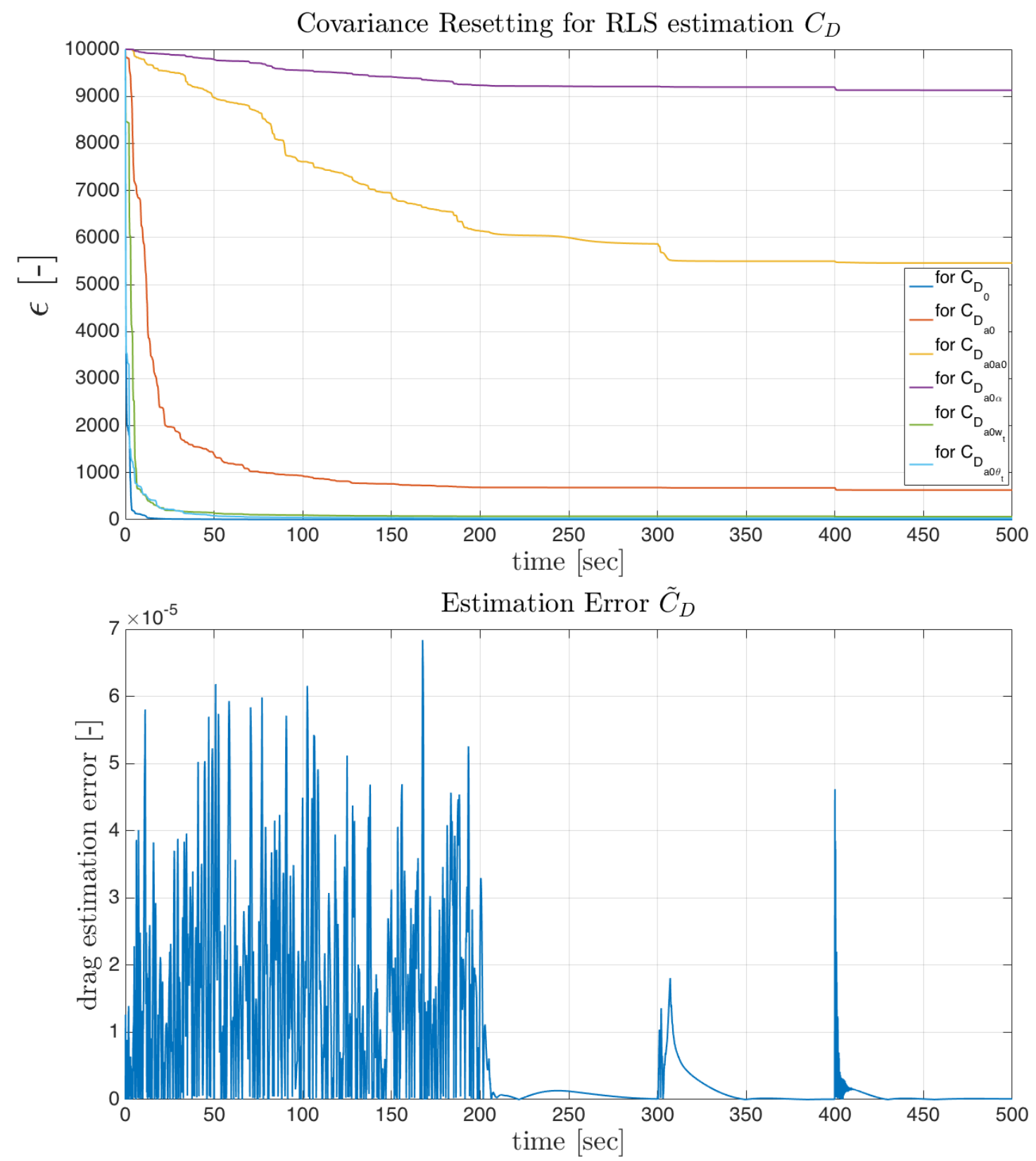

Figure 6. Covariance Resetting and Estimation Error Convergence for $C_{D}$ 

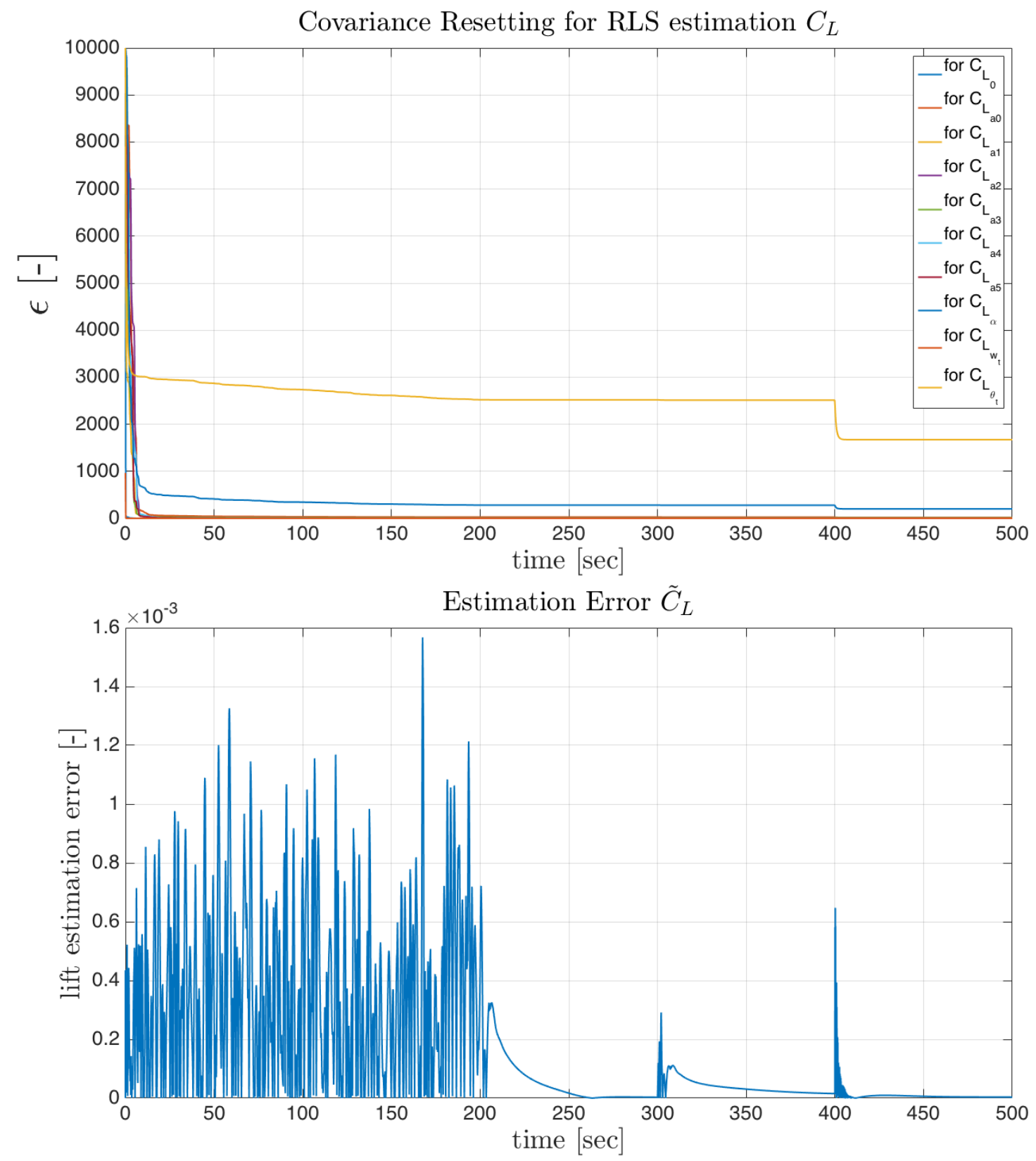

Figure 7. Covariance Resetting and Estimation Error Convergence for $C_{L}$ 

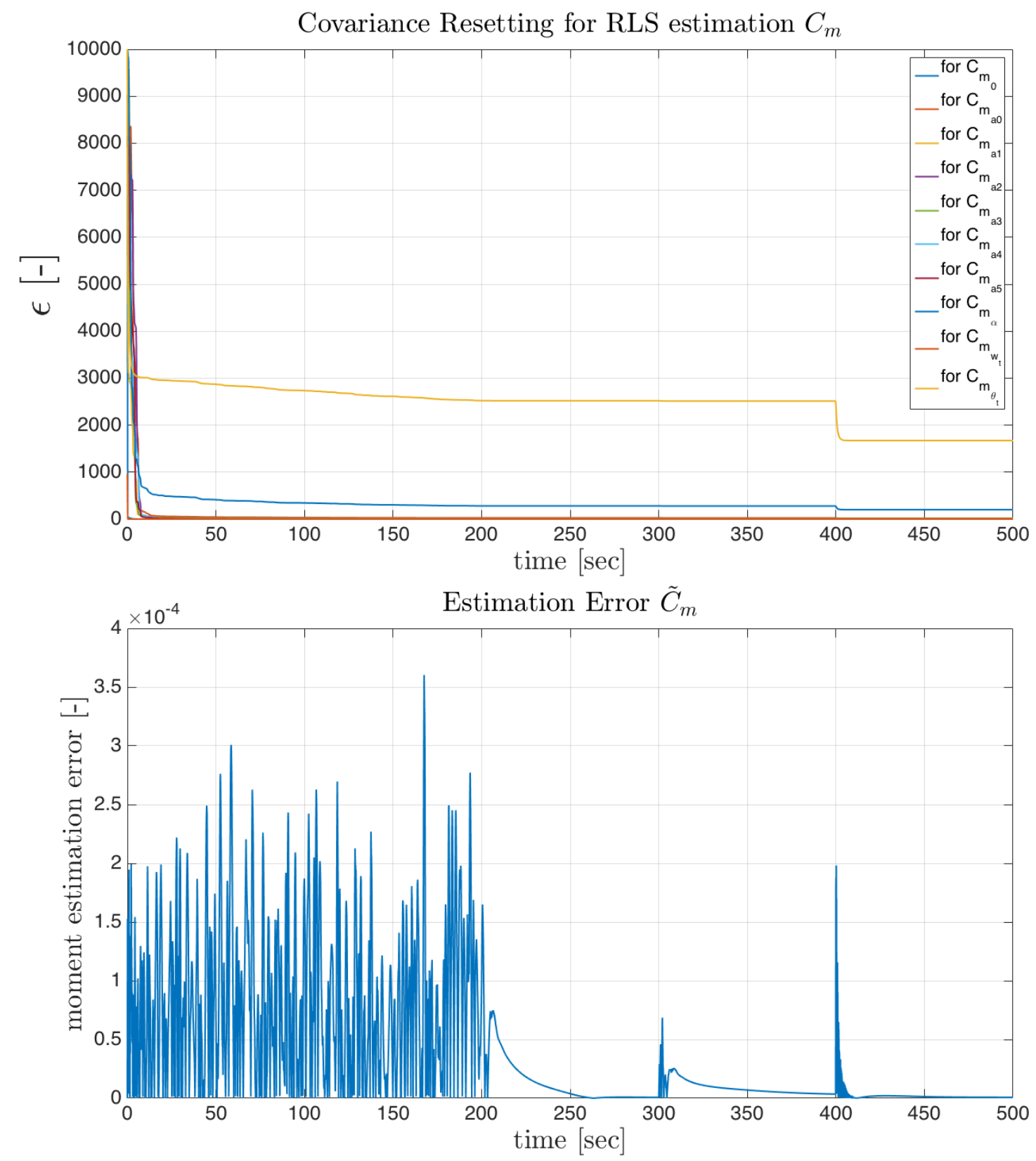

Figure 8. Covariance Resetting and Estimation Error Convergence for $C_{m}$ 


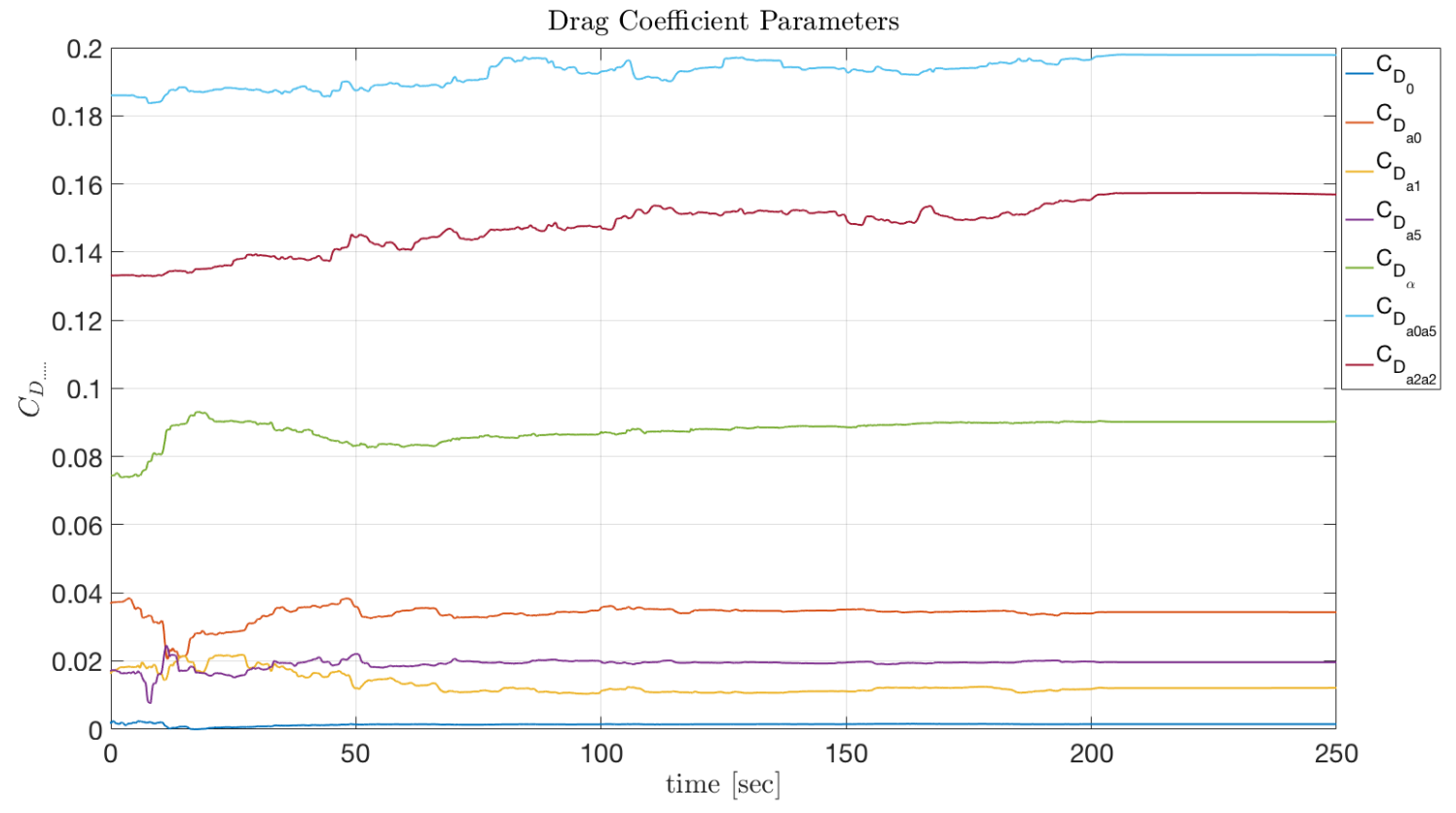

Figure 9. Real-time Drag Coefficient Parameter Estimation

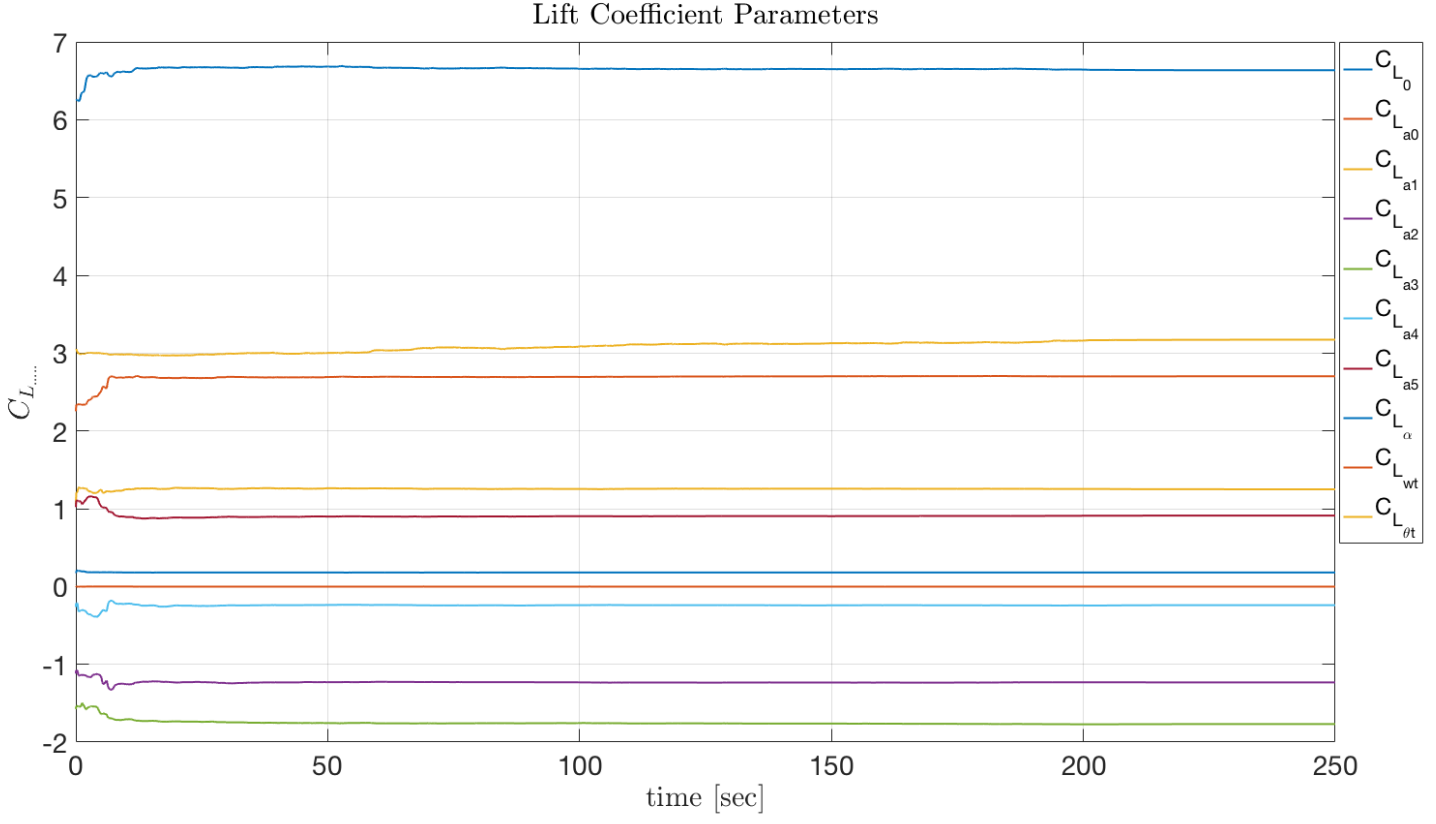

Figure 10. Real-time Lift Parameter Estimation 


\begin{tabular}{|c|c|c|c|}
\hline & Final Estimated Value & Truth Model Value & Estimation Error [\%] \\
\hline \hline$C_{L_{0}}$ & 0.1797 & 0.1788 & 0.5 \\
\hline$C_{L_{a_{0}}}$ & 2.6931 & 2.6910 & 0.1 \\
\hline$C_{L_{a_{1}}}$ & 1.2435 & 1.2385 & 0.4 \\
\hline$C_{L_{a_{2}}}$ & -1.2322 & -1.2486 & -1.3 \\
\hline$C_{L_{a_{3}}}$ & -1.7640 & -1.7727 & -0.5 \\
\hline$C_{L_{a_{4}}}$ & -0.2372 & -0.2295 & 3.4 \\
\hline$C_{L_{a_{5}}}$ & 0.9087 & 0.9232 & -1.6 \\
\hline$C_{L_{\alpha}}$ & 6.6519 & 6.6672 & -0.2 \\
\hline$C_{L_{w_{t}}}$ & -0.0013 & -0.0011 & 17.1 \\
\hline$C_{L_{\theta_{t}}}$ & 3.0984 & 2.9375 & 5.5 \\
\hline
\end{tabular}

Table 3. Lift Coefficient Parameter Estimation Value and Estimation Error

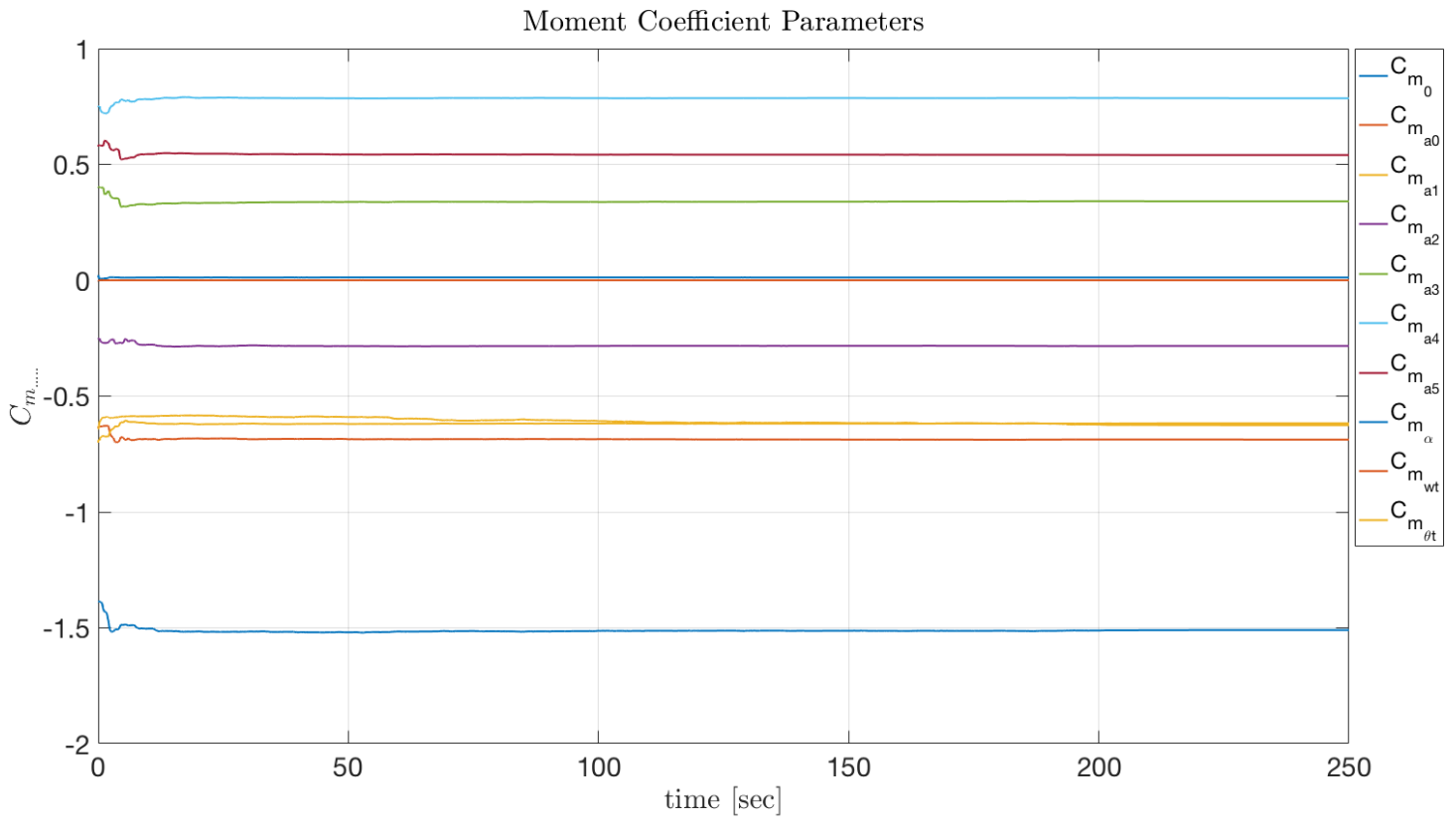

Figure 11. Real-time Moment Parameter Estimation

\begin{tabular}{|c|c|c|c|}
\hline & Final Estimated Value & Truth Model Value & Estimation Error [\%] \\
\hline \hline$C_{m_{0}}$ & 0.0123 & 0.0126 & -2.4 \\
\hline$C_{m_{a_{0}}}$ & -0.6848 & -0.6829 & 0.3 \\
\hline$C_{m_{a_{1}}}$ & -0.6154 & -0.6135 & 0.3 \\
\hline$C_{m_{a_{2}}}$ & -0.2840 & -0.2807 & 1.2 \\
\hline$C_{m_{a_{3}}}$ & 0.3392 & 0.3402 & -0.3 \\
\hline$C_{m_{a_{4}}}$ & 0.7847 & 0.7824 & 0.3 \\
\hline$C_{m_{a_{5}}}$ & 0.5411 & 0.5380 & 0.6 \\
\hline$C_{m_{\alpha}}$ & -1.5154 & -1.5236 & -0.5 \\
\hline$C_{m_{w_{t}}}$ & 0.0002 & 0.0001 & 11.1 \\
\hline$C_{m_{\theta_{t}}}$ & -0.6003 & -0.5471 & 9.7 \\
\hline
\end{tabular}

Table 4. Moment Coefficient Parameter Estimation Value and Estimation Error 
The drag coefficient in Table 5 includes the induced drag and a constant viscous drag $\left(C_{D_{v}}=0.01\right)$, but excludes the wave drag component. In Table 5, one drag count is equal to 0.0001.

\begin{tabular}{|c|c|c|}
\hline Variables & Clean Wing & Drag-Optimal Configuration with VCCTEF \\
\hline \hline$\alpha[\mathrm{deg}]$ & 2.5067 & 2.3156 \\
\hline$\delta[\mathrm{deg}]$ & 0 & $-1.5575 \leq \delta_{i, 3} \leq 3.5443$ \\
\hline$\delta_{e}[\mathrm{deg}]$ & -1.0541 & -1.8566 \\
\hline$w_{t}[\mathrm{ft}]$ & 3.0654 & 3.3722 \\
\hline$\theta_{t}[\mathrm{deg}]$ & 0.1238 & -0.1015 \\
\hline$C_{L}[-]$ & 0.4595 & 0.4595 \\
\hline$C_{m}[-]$ & -0.0042 & -0.0042 \\
\hline$C_{D}[$ drag count] & 186.03 & 183.14 \\
\hline
\end{tabular}

Table 5. Drag Optimization Results for VCCTEF Wing compared with Clean Wing Configuration

\section{Iterative Refinement Optimization}

The first iterative refinement optimization allows the RLS identification algorithm to update the aerodynamic parameters to a more accurate estimation. The updated optimal configuration results are given in Table 6. The optimal configuration changes slightly. Since the change in optimal drag is less than 0.00005 , additional iterative refinement optimization is not necessary. The results show that the aerodynamic parameters have already converged significantly during the parameter identification period.

\begin{tabular}{|c|c|c|}
\hline Variables & Original Optimal Configuration & Iterative Refinement Optimal Configuration \\
\hline \hline$\alpha[\mathrm{deg}]$ & 2.3156 & 2.3473 \\
\hline$\delta[\mathrm{deg}]$ & $-1.5575 \leq \delta_{i, 3} \leq 3.5443$ & $-1.6652 \leq \delta_{i, 3} \leq 3.3489$ \\
\hline$\delta_{e}[\mathrm{deg}]$ & -1.8566 & -1.8440 \\
\hline$w_{t}[\mathrm{ft}]$ & 3.3722 & 3.3637 \\
\hline$\theta_{t}[\mathrm{deg}]$ & -0.1015 & -0.0831 \\
\hline$C_{L}[-]$ & 0.4595 & 0.4595 \\
\hline$C_{m}[-]$ & -0.0042 & -0.0042 \\
\hline$C_{D}[$ drag count] & 183.14 & 183.17 \\
\hline
\end{tabular}

Table 6. Optimal Solution of Drag Minimization after Iterative Refinement Optimization

The results of the VCCTEF optimization after iterative refinement optimization are presented in Figs. 12 - 16. Figure 12 shows the optimized flap deflections from root to tip for all three flap segments. The trailing edge chordwise segments, $\delta_{3}$, have the largest deflections and the other two chordwise flaps segments follow in a circular-arc camber.

Figure 13 shows that the VCCTEF is able to adjust the wing shape to allow the aircraft to fly at a lower angle of attack for a specific lift coefficient. The lift-to-drag ratio distribution in Fig. 14 illustrates that the aircraft has a higher lift-to-drag ratio at the optimal angle of attack compared to the clean wing trim angle of attack. Figure 15 shows that the local lift distribution of the optimized VCCTEF configuration is driven towards the elliptical lift distribution. The drag polar in Fig. 16 shows that the VCCTEF is able to slightly decrease the drag for lift coefficients near the design lift coefficient in comparison with the clean wing configuration.

\section{Control Surfaces}

The time histories of the control surfaces deflections, $\delta$ and $\delta_{e}$, during the excitation simulation are illustrated in Figs. 17 and 18. The excitation of the VCCTEF for the RLS identification algorithm takes place the first 200 seconds and the iterative refinement optimization takes place at $t=300 \mathrm{~s}$. Between $302 \mathrm{~s} \leq t \leq 400 \mathrm{~s}$ the 


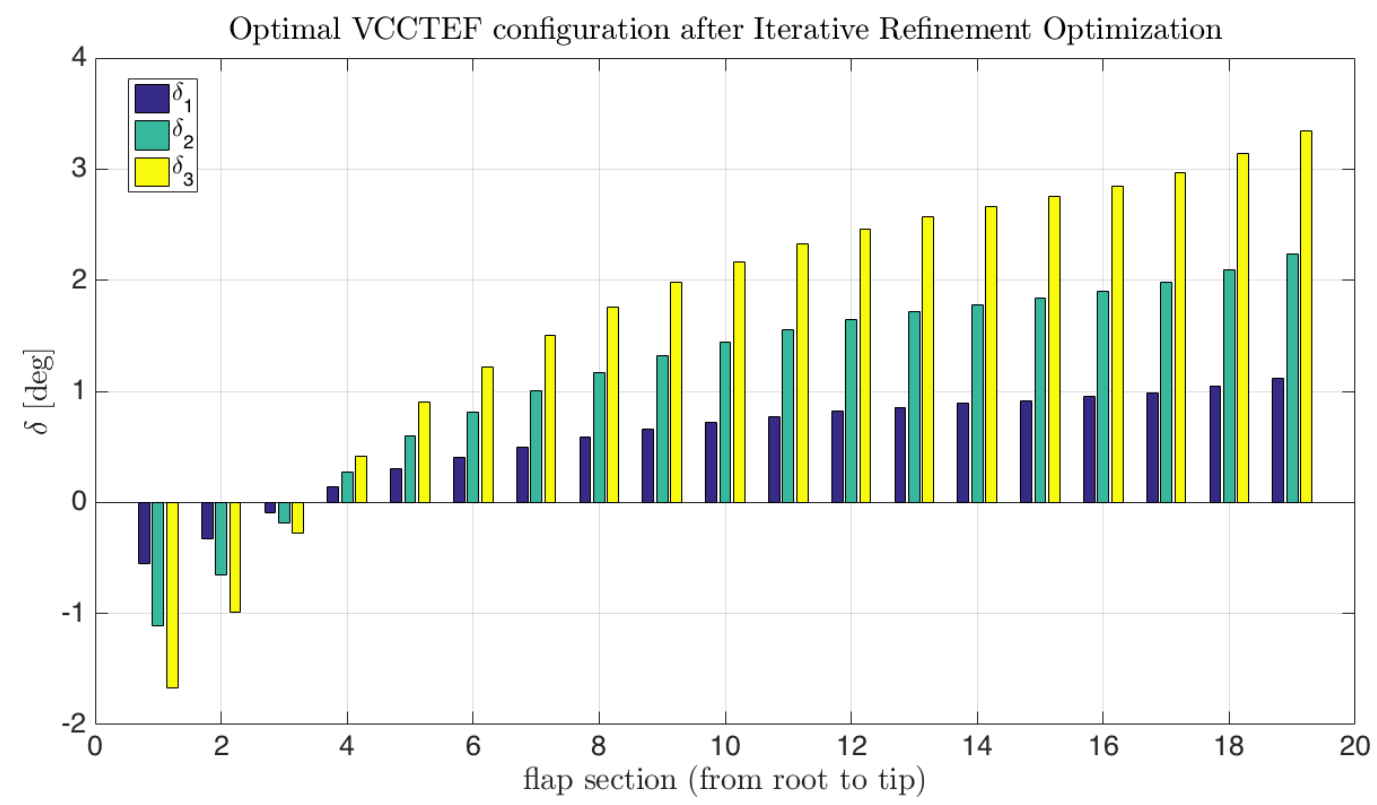

Figure 12. New Optimal VCCTEF Configurations after Iterative Refinement Optimization

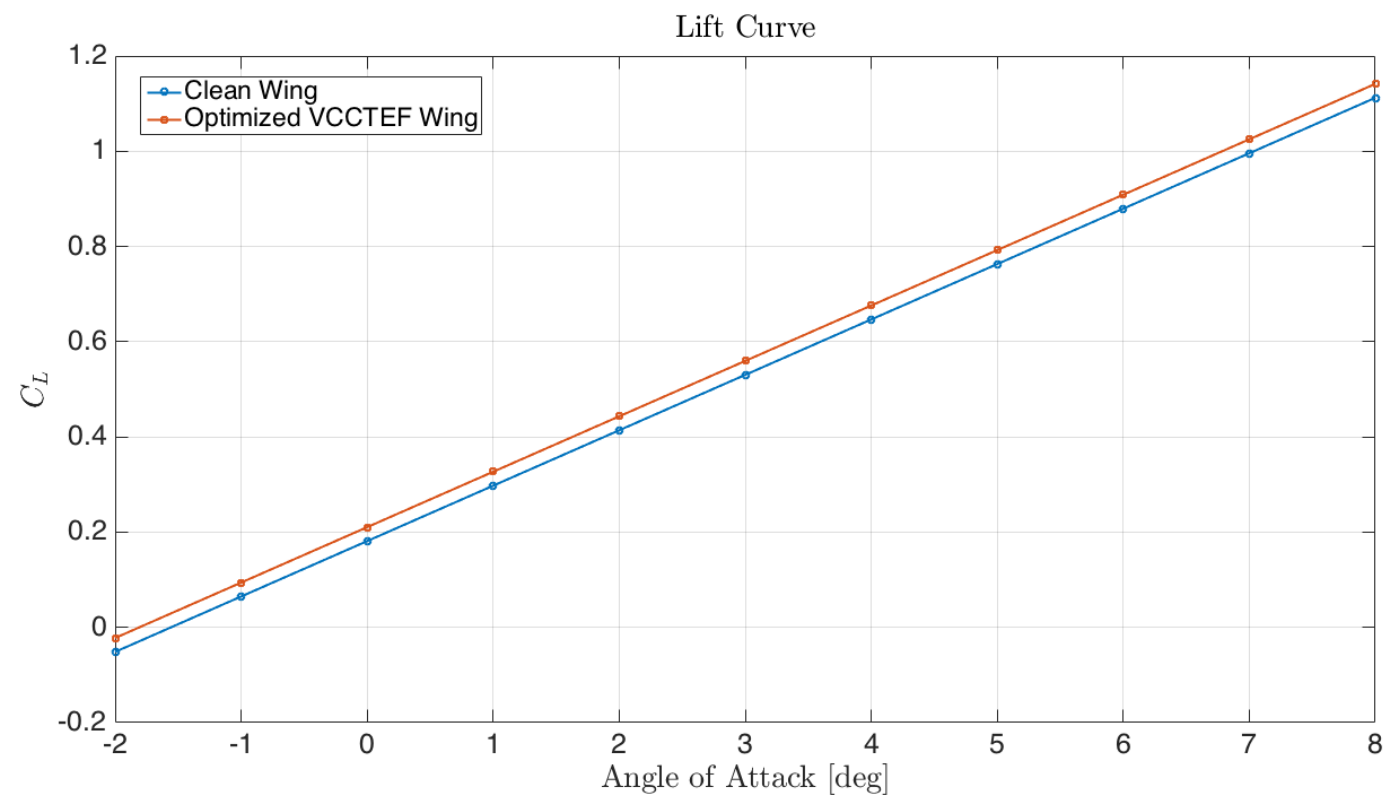

Figure 13. Lift Curve Comparison of Clean Wing Configuration and Optimized VCCTEF Configuration for Cruise Flight Condition 


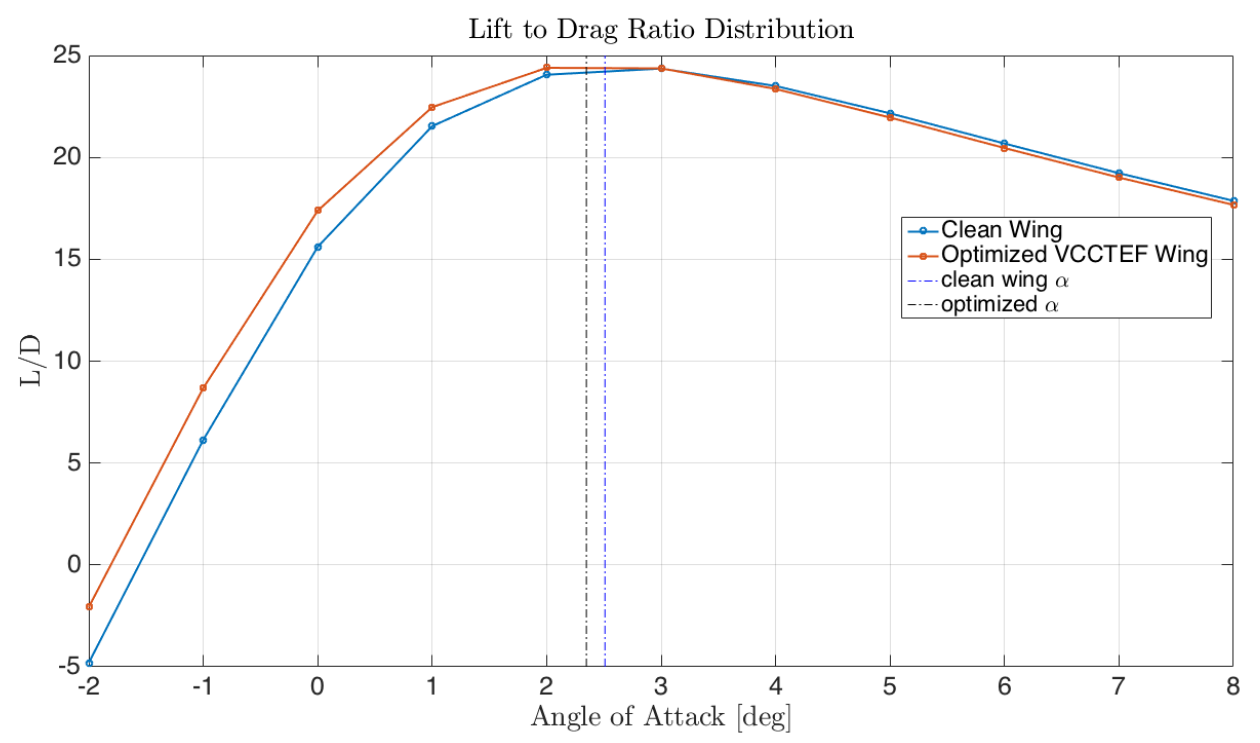

Figure 14. Lift to Drag Ratio Distribution of Clean Wing Configuration and Optimized VCCTEF Configuration for Cruise Flight Condition

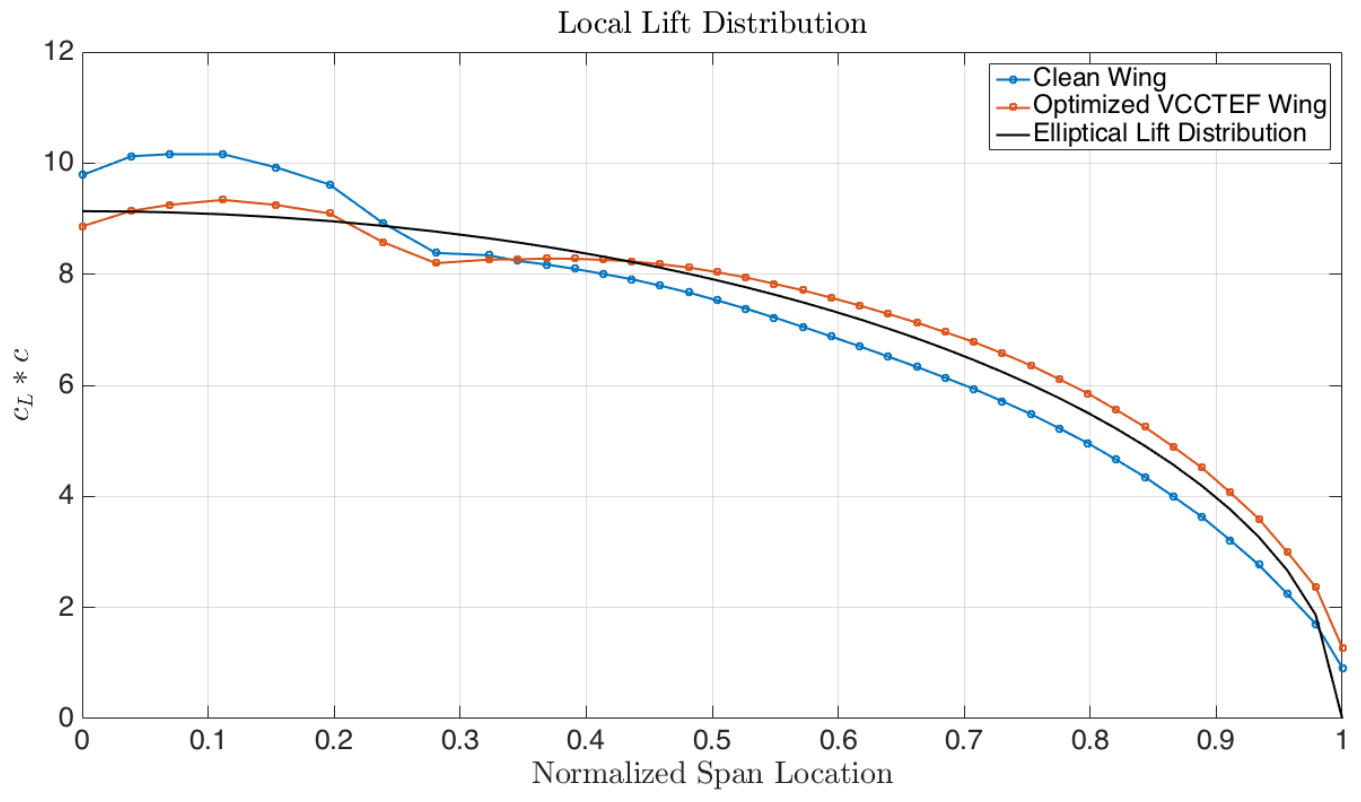

Figure 15. Lift Distribution of Clean Wing Configuration and Optimized VCCTEF Configuration for Cruise Flight Condition 


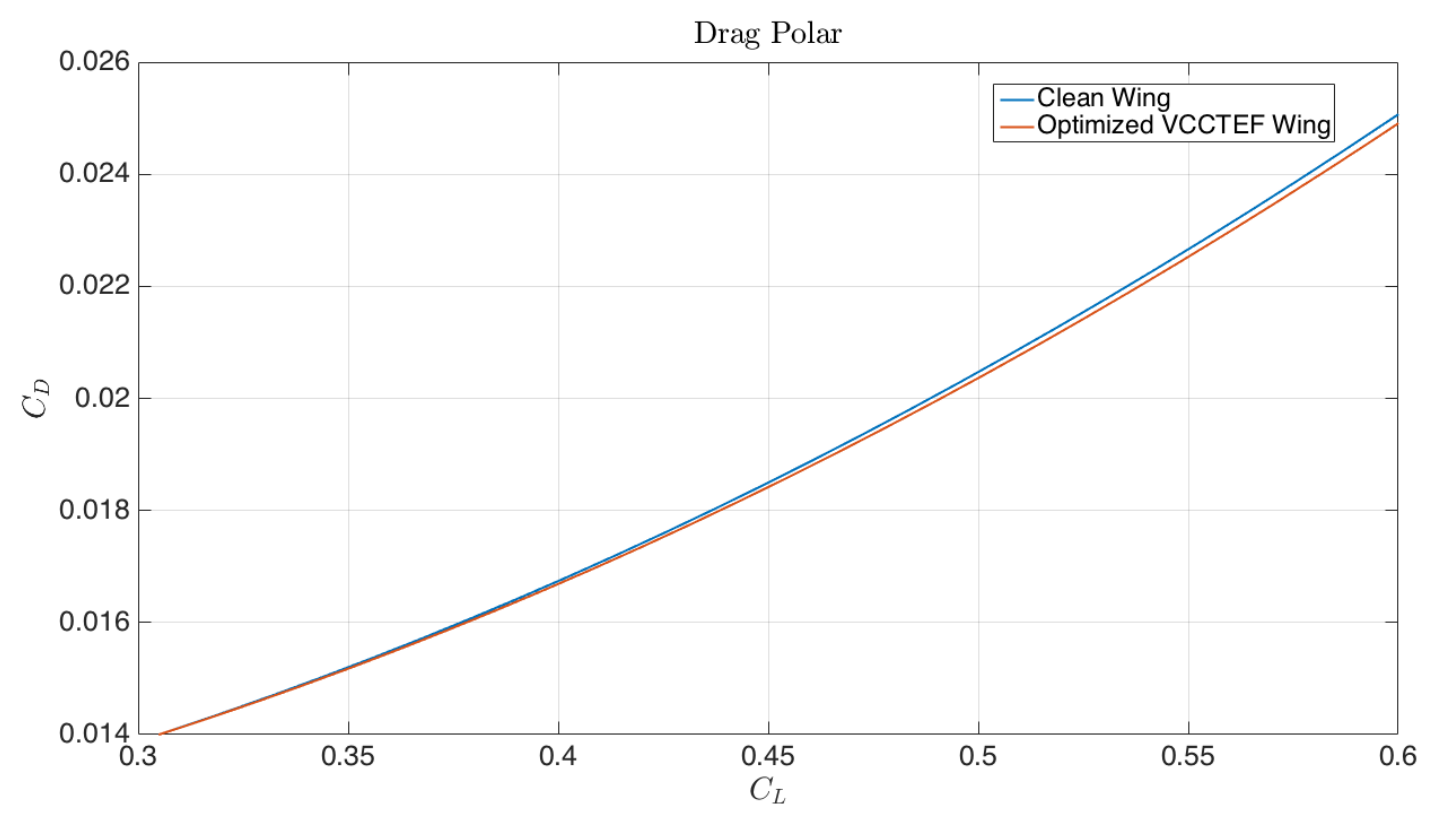

Figure 16. Drag Polar Comparison of Clean Wing Configuration and Optimized VCCTEF Configuration for Cruise Flight Condition

VCCTEF is deflected to clean wing configuration and between 400s $\leq t \leq 500$ s the VCCTEF is deflected to the optimal wing configuration. The elevator responds accordingly.

In Fig. 18 the calculated optimal elevator deflection is illustrated by the dotted orange line for the last 100 seconds of the simulation. The actual elevator deflection corresponds closely with this calculated optimal elevator deflection. The difference between the actual elevator deflection and the calculated optimal elevator deflection is $0.006^{\circ}$.

\section{Flight Dynamic States}

The nonlinear rigid body flight dynamic and aeroelastic states of the GTM aircraft are shown in Figs. 19 and 20. From these figures, we can see that the perturbation of the VCCTEF excites $\alpha, w_{t}$ and $\theta_{t}$ significantly. This is important, since these states need to be excited thoroughly to allow for the RLS identification.

The linear altitude-hold flight controller has difficulties to hold the altitude at the excitation period, but succeeds in bringing the aircraft back to the correct altitude during clean wing and optimal VCCTEF configuration. The flight path angle varies during the excitation period, resulting in changes to the airspeed during the excitation period. After the excitation period, the states converge back to their clean wing trim or optimal VCCTEF trim states.

The dotted orange lines in Figs. 19 and 20 show the calculated optimal values for the $\alpha, w_{t}, \theta_{t}$ during the last 100 seconds of the simulation. The figures show that these states correspond closely with their calculated optimal values.

\section{Aerodynamic Coefficients}

Figure 21 shows the aerodynamic coefficients during the simulation. The excitation of the VCCTEF is clearly visible during the first 200 seconds. The aerodynamic coefficients vary significantly during this excitation period. This is important since it allows for better identification in the RLS identification algorithm.

From Fig. 21 one can see that the drag coefficient indeed converges to the calculated optimal value $(400 \mathrm{~s} \leq t \leq 500 \mathrm{~s})$, and is lower than the value at the clean wing configuration $(300 \mathrm{~s} \leq t \leq 400 \mathrm{~s})$. The final drag, lift and moment coefficients in the simulation are very close to the calculated optimal values, with a small difference of $0.00004,0.001$ and 0.00001 respectively. This difference is due to the dissimilarity between the estimated aerodynamic parameters and the "true" aerodynamic parameters and the slight deviation of the actual elevator deflection in comparison with the calculated optimal elevator deflection. 


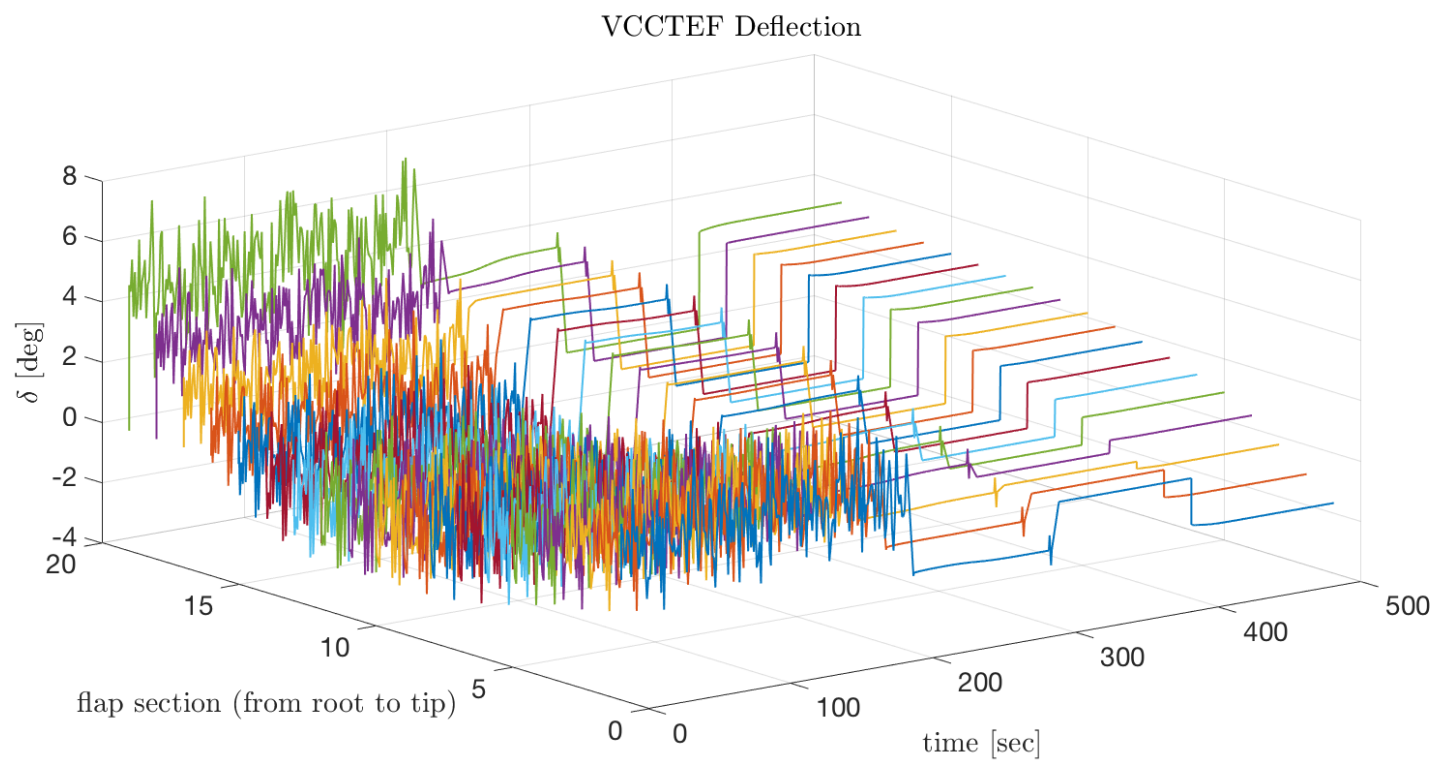

Figure 17. VCCTEF Deflections

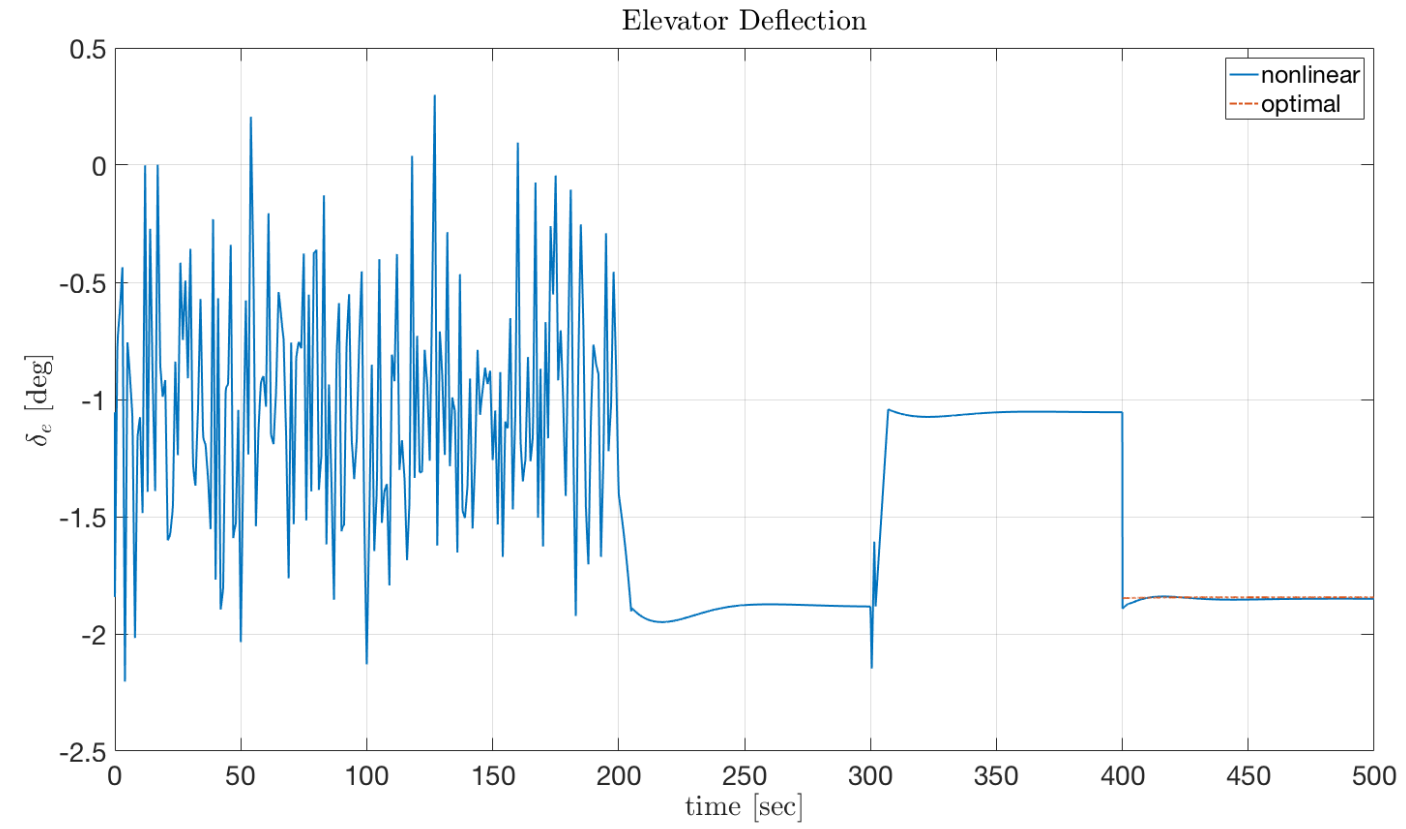

Figure 18. Elevator Deflection 

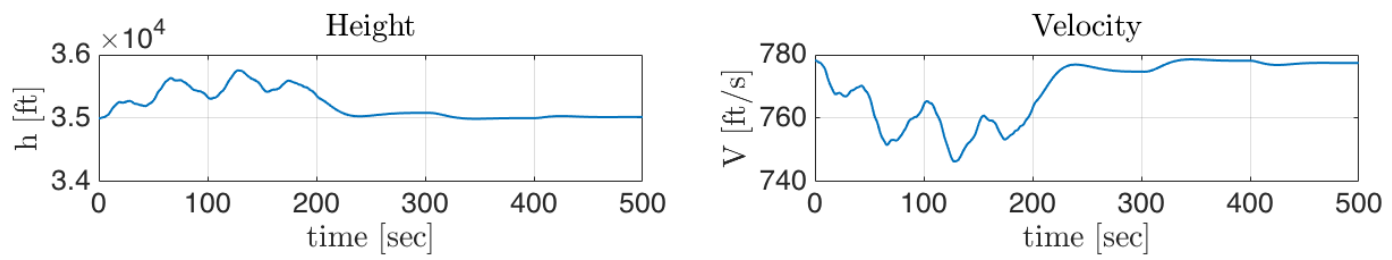

Angle of Attack

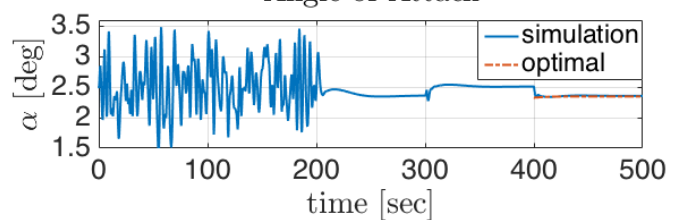

Flight Path Angle

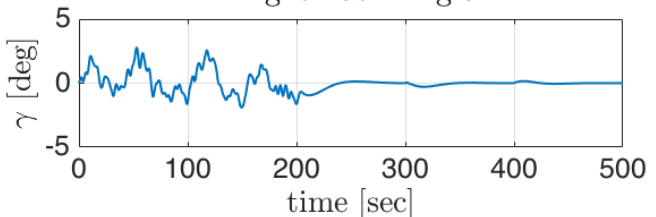

Pitch Angle

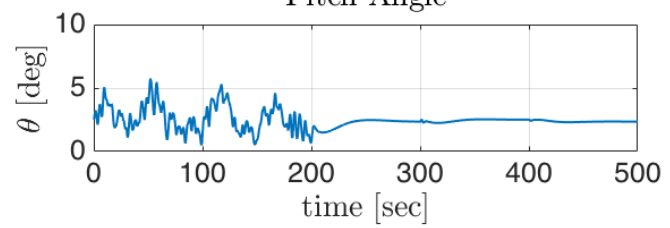

Pitch Rate

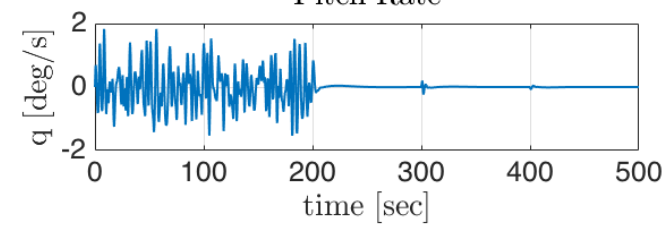

Figure 19. Nonlinear Rigid Body Flight Dynamic States
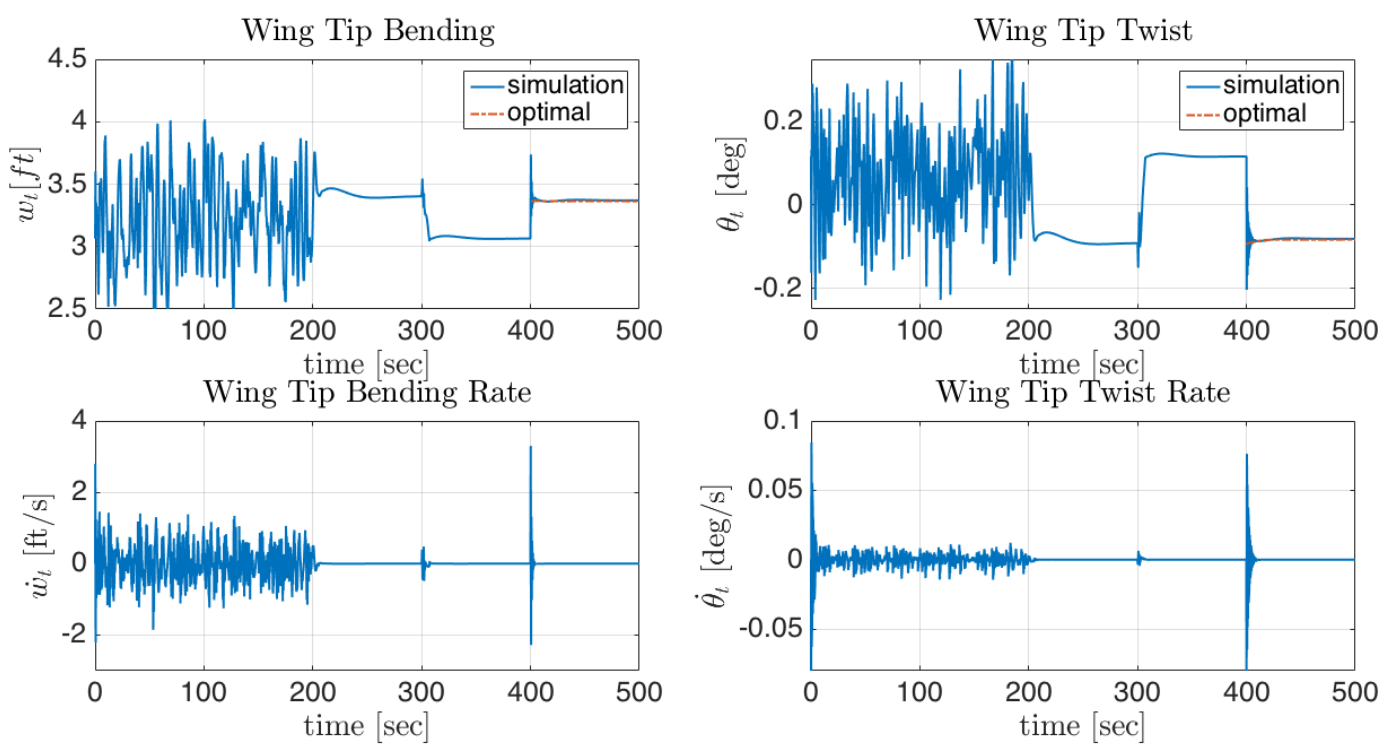

Figure 20. Nonlinear Aeroelastic Flight Dynamic States 

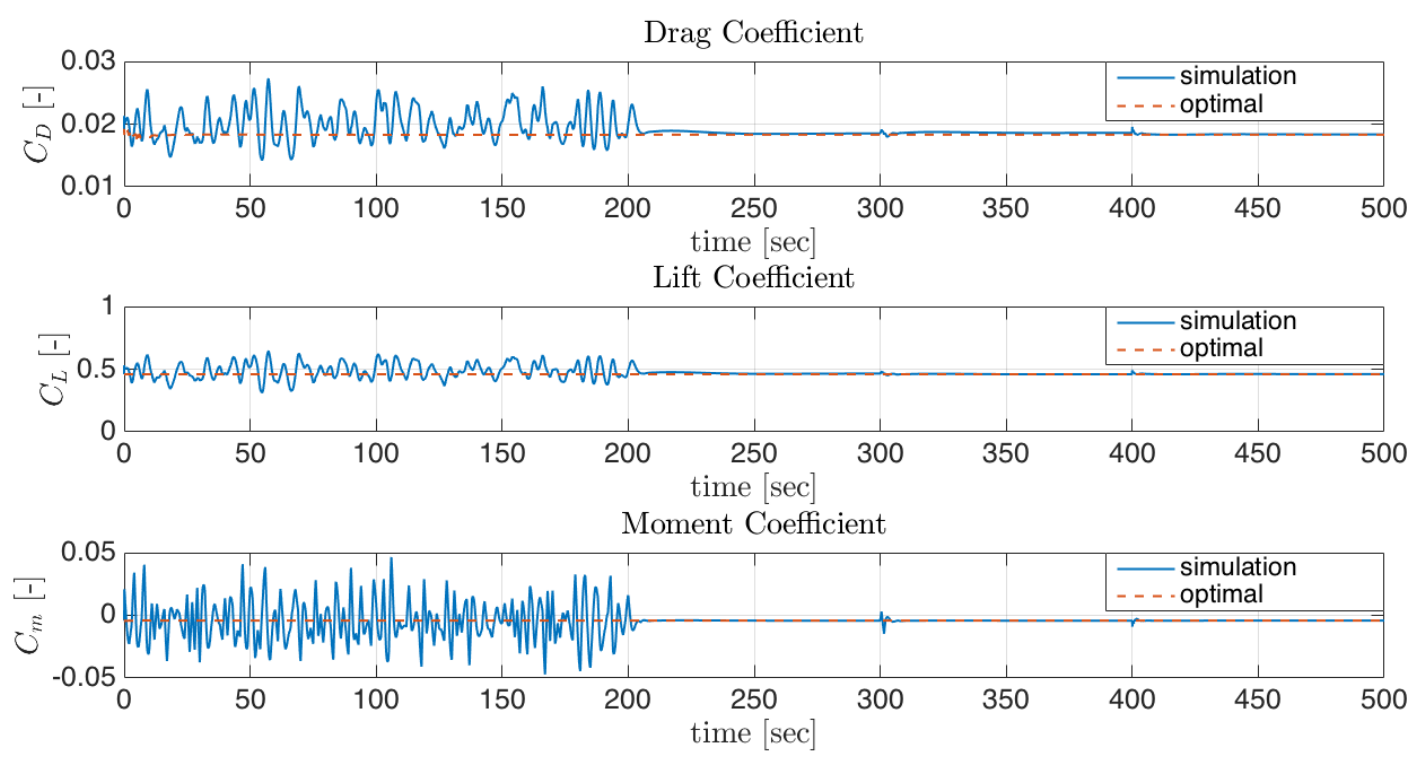

Figure 21. Aerodynamic Coefficients

\section{Discussion}

It is important to qualify the limitations and assumptions in the model used for this study that may affect the drag reduction assessments. First of all, the AVL model used in this study is an inviscid code not capable of directly calculating viscous drag and wave drag contributions. A constant viscous drag component is added in the simulation. In future studies, the effect of viscous drag and wave drag will be considered.

Secondly, the spanwise deflections of the VCCTEF are constrained to a fifth-degree Chebyshev polynomial. From the optimization results in Fig. 12 this shape function seems sufficient, however a consideration would be to model the inboard and outboard flap sections of the VCCTEF with two separate polynomials. The optimal number of spanwise flaps and the shape of the cambered flap deflection should also be considered, especially when viscous and transonic effects are incorporated in the drag model. Future work on the design of the VCCTEF will include studies of the optimum shape between the individual flap sections, the optimum number of spanwise flaps, and the optimum shape between the chordwise flap segments. The optimization study for the number of spanwise flaps should be a trade study that includes the weight of the flap sections.

Thirdly, in the recursive least squares approach the Mach number is assumed to stay constant $(M=0.8)$, although during the simulation the Mach number varied slightly $(0.78 \leq M \leq 0.80)$. The effect of the Mach number on the aerodynamic coefficient parameters could be assessed similarly as is done in the modeling of the truth model (see Eqs. 48 to 55). If the Mach number should be considered in the RLS identification algorithm, the excitation of the estimated parameters should allow for enough varying input with respect to the Mach number. This could be studied in future research on the RLS identification algorithm, although from an operational consideration it is not practical to change the airspeed during cruise.

The recursive least squares algorithm is not able to accurately identify the aerodynamic parameters with respect to the wing tip bending $w_{t}$. Further research should be done to improve the identification of all the aerodynamic coefficient parameters.

Furthermore, the effect of sensor noise in the aerodynamic parameters and estimated variables on the RLS identification algorithm is not taken into consideration in this study. This would be an important consideration for further studies.

It should be noted that the proposed adaptive real-time drag minimization strategy will depend largely on sensor technologies that would enable in-flight drag measurements. If the aircraft is in trim, the engine thrust and the aerodynamic drag force are in balance. Thus, the drag force can be inferred from the engine thrust. In practice, engine thrust is not a quantity that could be measured directly. For certain commercial 
engines, fuel flow meters are available and could be used to estimate engine thrust via calibration. Other forms of engine thrust measurements could be considered such as strain gauges and load sensors which can be calibrated with engine static thrust to provide a relationship for estimating in-flight engine thrust. Since the wing shape has a profound impact on drag, it is a possibility that future innovative sensors could be developed to measure the in-flight wing shape deflection directly. The wing shape could be calibrated with aerodynamic drag measurements in wind tunnel tests or drag computation from CFD to provide a means for in-flight drag estimation. NASA has recently developed a fiber-optic shape sensor (FOSS) technology which could be used in this application.

\section{Conclusions}

The introduction of lightweight modern materials into the airframe structure, with the goal of lowering the aircraft weight and consequently reducing fuel consumption, has increased the flexibility of modern aircraft wings. This flexibility may negatively impact the wing aerodynamic characteristics by altering the optimal wing shape undesirably when the wings are under load. This potential negative impact could be alleviated by actively shaping the wings in order to achieve desirable aerodynamic characteristics. One way of achieving this wing shaping is through a flap system such as the variable camber continuous trailing edge flap (VCCTEF). The VCCTEF configuration can be optimized using various optimization methods to minimize drag at different cruise conditions. By doing this, the flexibility is exploited to control the shape of the wing during flight and consequently restore the aerodynamic efficiency.

This study involves determining the optimal VCCTEF configuration for minimum drag in real-time during cruise conditions for the NASA Generic Transport Model (GTM) aircraft. The Athena Vortex Lattice (AVL) method is used as the aircraft aerodynamic modeling tool. This aerodynamic model is approximated by a truth model. The truth model shows good agreement with the AVL model with average errors below $1 \%$ for all aerodynamic coefficients and local coefficient distributions.

A recursive least-squares identification algorithm is built to estimate the aerodynamic parameters in realtime. The estimation errors show that this approach can give accurate approximations for the aerodynamic coefficients. The percentage errors of the estimation of the aerodynamic coefficient parameters are below $16 \%, 6 \%$ and $10 \%$ percent for the drag, lift and moment parameters, respectively, with the exception of the parameters with respect to $w_{t}$.

Iterative refinement optimization is used to update the estimated coefficients by perturbing the VCCTEF close to the previously found optimal solution to identify an improved optimal solution. This approach can be effective in identifying a new optimum in a close proximity to the previous calculated optimal solution.

The drag minimization algorithm is able to find the optimal VCCTEF configuration, while conforming to several constraints. The optimal VCCTEF is able to reduce the induced drag by 2.9 drag count in comparison with a clean wing configuration at the design cruise condition. The optimal VCCTEF configuration drives the local lift distribution towards the more aerodynamically efficient elliptical lift distribution.

The simulation study of the real-time adaptive least-squares drag minimization shows the excitation of the states and the convergence of the states to the minimum drag configuration. The linear controller is not able to hold the altitude during the excitation period, however succeeds in stabilizing the aircraft after the excitation. A nonlinear controller may provide a better altitude-hold flight control mode, but with an increase in complexity. The simulation shows that the $\alpha, w_{t}$ and $\theta_{t}$ converge closely to their calculated optimal values when the VCCTEF is deflected to the optimal configuration. From this, we can conclude that these states follow the relations as defined in the constraints of the drag minimization algorithm. The aerodynamic coefficients are excited significantly during the excitation period and converge to their optimal values when the VCCTEF is deflected at the calculated optimal configuration.

In previous studies it is shown that the VCCTEF can reduce drag significantly at off design cruise conditions. ${ }^{3}$ The current study shows that the VCCTEF is also able to reduce drag at design cruise conditions. We can conclude that active wing shaping through the VCCTEF is a promising solution to minimize drag during flight at both design and off-design cruise conditions.

\section{Acknowledgements}

The authors would like to thank the Fixed Wing / Advanced Air Transport Technology Project under the Advanced Air Vehicles Program of NASA Aeronautics Research Mission Directorate (ARMD) for funding 
support of this work.

\section{References}

\footnotetext{
${ }^{1}$ Nguyen, N., "Elastically Shaped Future Air Vehicle Concept," NASA Innovation Fund Award 2010 Report, October 2010, Submitted to NASA Innovative Partnerships Program, http://ntrs.nasa.gov/archive/nasa/casi.ntrs.nasa.gov/20110023698.pdf ${ }^{2}$ Nguyen, N. and Urnes, J., "Aeroelastic Modeling of Elastically Shaped Aircraft Concept via Wing Shaping Control for Drag Reduction," AIAA Atmospheric Flight Mechanics Conference, AIAA-2012-4642, August 2012.

${ }^{3}$ Lebofsky, S., Ting, E., and Nguyen, N., "Aeroelastic Modeling and Drag Optimization of Flexible Wing Aircraft with Variable Camber Continuous Trailing Edge Flap," 32nd AIAA Applied Aerodynamics, AIAA 2014-2443, June 2014.

${ }^{4}$ Kaul, U. and Nguyen, N., "Drag Optimization Study of Variable Camber Continuous Trailing Edge Flap (VCCTEF) Using OVERFLOW," 32nd AIAA Applied Aerodynamics, AIAA 2014-2444, June 2014.

${ }^{5}$ Drela, M., and Youngren, H., AVL 3.26 User Primer, MIT Aero/Astro, Aerocraft, Inc., Cambridge, MA, 2006.

${ }^{6}$ Theodorsen, T., "General Theory of Aerodynamic Instability and the Mechanism of Flutter," NACA-TR-496, January 1949.

${ }^{7}$ Basappa and Jategaonkar, R.V., "Evaluation of Recursive Methods for Aircraft Parameter Estimation," AIAA Atmospheric Flight Mechanics Conference and Exhibit, AIAA 2004-5063, August 2004.

${ }^{8} \mathrm{Su}$, W., Reich, G., "Modeling of Artificial Hair Sensors for Vibration Control of Flexible Wings," AIAA/ASCE/AHS/ASC Structures, Structural Dynamics, and Materials Conference, AIAA-2016-1958, January 2016.

${ }^{9}$ Nguyen, N., Trinh, K., Reynolds, K., Kless, J., Aftosmis, M., Urnes, J., and Ippolito, C., "Elastically Shaped Wing Optimization and Aircraft Concept for Improved Cruise Efficiency," AIAA Aerospace Sciences Meeting, AIAA-2013-0141, January 2013.

${ }^{10}$ Boeing Report No. 2012X0015, "Development of Variable Camber Continuous Trailing Edge Flap System," October 4, 2012.

${ }^{11}$ Urnes, J., Nguyen, N., Ippolito, C., Totah, J., Trinh, K., and Ting, E., "A Mission Adaptive Variable Camber Flap Control System to Optimize High Lift and Cruise Lift to Drag Ratios of Future N+3 Transport Aircraft," AIAA Aerospace Sciences Meeting, AIAA-2013-0214, January 2013.

${ }^{12}$ Nguyen, N, Precup, N., Urnes, J., Nelson, C., Lebofsky, S., Ting, E., and Livne, E., "Experimental Investigation of a Flexible Wing with a Variable Camber Continuous Trailing Edge Flap Design," 32nd AIAA Applied Aerodynamics Conference, AIAA-2014-2442, June 2014.

${ }^{13}$ Nguyen, N., Ting, E., Nguyen, D., Trinh, K., "Flutter Analysis of Mission-Adaptive Wing with Variable Camber Continuous Trailing Edge Flap," 55th AIAA/ASME/ASCE/AHS/ASC Structures, Structural Dynamics, and Materials Conference, AIAA-2014-0839, January 2014.

${ }^{14}$ Nguyen, N., Ting, E., Nguyen, D., and Trinh, K., "Flight Dynamic Modeling and Stability Analysis of Flexible Wing Generic Transport Aircraft," 55th AIAA/ASME/ASCE/AHS/ASC Structures, Structural Dynamics, and Materials Conference, AIAA-2014-1040, January 2014.

${ }^{15}$ Tal, E., Nguyen, N. and Ting, E., "Comparison of Unsteady Aerodynamics Approximations for Time-Domain Representation of Frequency-Independent Aeroelastic State-Space Models," 56th AIAA/ASME/ASCE/AHS/ASC Structures, Structural Dynamics, and Materials Conference, January 2015.

${ }^{16}$ Nguyen, N., Swei, S., and Ting, E., "Adaptive Linear Quadratic Gaussian Optimal Control Modification for Flutter Suppression of Adaptive Wing," AIAA Infotech@Aerospace Conference, AIAA 2015-0118, January 2015.

${ }^{17}$ Kokotovic, P., Khalil, H., and O'Reilly, J., Singular Perturbation Methods in Control: Analysis and Design, Society for Industrial and Applied Mathematics, 1987.

${ }^{18}$ Ardema, M., "Computational Singular Perturbation Method for Dynamical Systems", AIAA Journal of Guidance, Control, and Dynamics, Vol. 14, 661-663, 1981.

${ }^{19}$ Ippolito C. and Nguyen, N., "A Preliminary Study for Optimal Longitudinal-Mode Flight Control through Distributed Aeroelastic Shaping," 55th AIAA/ASME/ASCE/AHS/ASC Structures, Structural Dynamics, and Materials Conference, AIAA2014-1044, January 2014.

${ }^{20}$ Brown, N. and Schaefer, J., "Peak-Seeking Optimization of Trim for Reduced Fuel Consumption: Flight-Test Results," AIAA Guidance, Navigation, and Control Conference, AIAA-2013-5171, August 2013.

${ }^{21}$ Nobbs, S.G., "Development of the Full-Envelope Performance Seeking Control Algorithm," 28th AIAA Joint Propulsion Conference, AIAA-1992-3748, July 1992.

${ }^{22}$ Nguyen, N. and Tal, E., "A Multi-Objective Flight Control Approach for Performance Adaptive Aeroelastic Wing," 56th AIAA/ASCE/AHS/ASC Structures, Structural Dynamics, and Materials Conference (AIAA Science and Technology Forum), January 2015.
} 